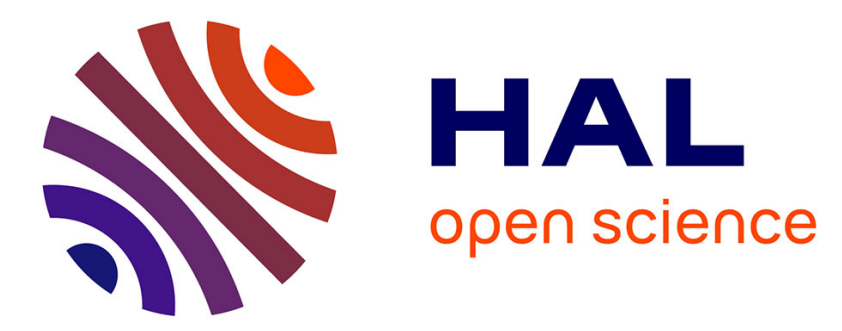

\title{
MineRank: Leveraging users' latent roles for unsupervised collaborative information retrieval
}

Laure Soulier, Lynda Tamine, Chirag Shah

\section{To cite this version:}

Laure Soulier, Lynda Tamine, Chirag Shah. MineRank: Leveraging users' latent roles for unsupervised collaborative information retrieval. Information Processing and Management, 2016, 52 (6), pp.11221141. 10.1016/j.ipm.2016.05.002 . hal-01353589

\section{HAL Id: hal-01353589 \\ https://hal.sorbonne-universite.fr/hal-01353589}

Submitted on 12 Aug 2016

HAL is a multi-disciplinary open access archive for the deposit and dissemination of scientific research documents, whether they are published or not. The documents may come from teaching and research institutions in France or abroad, or from public or private research centers.
L'archive ouverte pluridisciplinaire HAL, est destinée au dépôt et à la diffusion de documents scientifiques de niveau recherche, publiés ou non, émanant des établissements d'enseignement et de recherche français ou étrangers, des laboratoires publics ou privés. 


\title{
MineRank: Leveraging Users' Latent Roles for Unsupervised Collaborative Information Retrieval
}

\author{
Laure Soulier*, Lynda Tamine** and Chirag Shah*** \\ * Sorbonne Universités, UPMC Univ Paris 06, \\ UMR 7606, LIP6, F-75005, Paris,France \\ ** Toulouse University UPS IRIT \\ 118 route de Narbonne, 31062 Toulouse Cedex 9 \\ *** School of Communication \& Information (SC\&I) \\ Rutgers University \\ 4 Huntington St, New Brunswick, NJ 08901, USA
}

Email address: laure.soulier@lip6.fr*, tamine@irit.fr** and chirags@rutgers.edu*** (Laure Soulier*, Lynda Tamine** and Chirag Shah ${ }^{* * *}$ ) 


\title{
MineRank: Leveraging Users' Latent Roles for Unsupervised Collaborative Information Retrieval
}

\author{
Laure Soulier*, Lynda Tamine** and Chirag Shah*** \\ * Sorbonne Universités, UPMC Univ Paris 06, \\ UMR 7606, LIP6, F-75005, Paris,France \\ ** Toulouse University UPS IRIT \\ 118 route de Narbonne, 31062 Toulouse Cedex 9 \\ *** School of Communication \& Information (SC\&I) \\ Rutgers University \\ 4 Huntington St, New Brunswick, NJ 08901, USA
}

\begin{abstract}
Research on collaborative information retrieval (CIR) has shown positive impacts of collaboration on retrieval effectiveness in the case of complex and/or exploratory tasks. The synergic effect of accomplishing something greater than the sum of its individual components is reached through the gathering of collaborators' complementary skills. However, these approaches often lack the consideration that collaborators might refine their skills and actions throughout the search session, and that a flexible system mediation guided by collaborators' behaviors should dynamically adapt to this situation in order to optimize search effectiveness. In this article, we propose a new unsupervised collaborative ranking algorithm which leverages collaborators' actions for (1) mining their latent roles in order to extract their complementary search behaviors; and (2) ranking documents with respect to the latent role of collaborators. Experiments using two user studies with respectively 25 and 10 pairs of collaborators demonstrate the benefit of such an unsupervised method driven by collaborators' behaviors throughout the search session. Also, a qualitative analysis of the identified latent role is proposed to explain an over-learning noticed in one of the datasets.
\end{abstract}

Keywords: Collaborative information retrieval, unsupervised role mining,

Email address: laure.soulier@lip6.fr*, tamine@irit.fr** and chirags@rutgers.edu*** (Laure Soulier*, Lynda Tamine** and Chirag Shah***) 
latent role, user study

\section{Introduction}

In recent years, researchers have argued that in addition to creating better algorithms and systems for individualized search and retrieval, a substantial leap can be taken by incorporating collaborative aspects in Information Retrieval (IR) (Twidale et al., 1997), referred to as Collaborative Information Retrieval (CIR) (Fidel et al., 2000). However, simply allowing multiple people collaborate on a search task does not guarantee any advantages over a single searcher. To gain advantages, one needs to look deeper into the aspects of collaboration that make it successful and investigate how those aspects can be incorporated in a search setting. Many have found that when the collaborators bring a diverse set of skills to a project, they could achieve something more than what they could using their individual skills and contributions (Soulier et al., 2014). But how does one ensure the use of such diverse skills in search? One approach could be asking the searchers involved in a CIR project about the roles (e.g., query constructor, information assessor) they would like to play. However, these collaborators may either not know about such skills they have or may be unable to specify any preferences. Therefore, one may need to automatically mine their skills through behavioral features. Recently, this approach (Soulier et al., 2014) has been proposed, aiming at dynamically identifying, through search features, the possible roles of collaborators according to a role taxonomy (Golovchinsky et al., 2009). However, the labeled roles are predefined regardless of the users, and accordingly, one shortcoming of the proposed approach is its inability to ensure that the identified roles exactly fit with collaborators' search skills.

To tackle this gap, this current article presents a new approach - called MineRank - that mines in real time the unlabeled role that collaborators play in a CIR context. The objective is to leverage the diversity of the collaborators' search skills in order to ensure the division of labor policy and to optimize the overall performance. Instead of following predefined labels or a taxonomy of roles, MineRank is an unsupervised algorithm that (1) learns about the complementarity of collaborators' unlabeled roles in an unsupervised manner using various search behavior-related 
features for each individual involved, and (2) re-injects these unlabeled roles to collaboratively rank documents. The algorithm is used for various experiments and evaluated using retrieval effectiveness at various levels. The results show that the model is able to achieve synergic effect in CIR by learning the latent role of collaborators.

The remainder of the article is structured as follows. Section 2 presents the related work. In Section 3, we motivate our approach and introduce the problem definition. Section 4 focuses on our two-step unsupervised CIR model relying on the collaborators' unlabeled roles. The experimental evaluation and results are described in Section 5. Section 6 concludes the article.

\section{Related Work}

\subsection{Collaborative Information Retrieval}

Collaborative information retrieval (CIR) is defined as a search process that involves multiple users solving a shared information need (Golovchinsky et al., 2009). Research has found that this setting is particularly beneficial in the case of complex or exploratory information tasks (Morris and Horvitz, 2007 ) in which a lone individual would suffer from insufficient knowledge or skills. Indeed, collaboration in search could improve in retrieval effectiveness by providing the opportunity to gather complementary skills and/or knowledge in order to solve an information need, as well as addressing mutual benefits of collaborators through the synergic effect of the collaboration (Shah and González-Ibáñez, 2011b).

Collaboration between users is supported by three main principles: (1) avoiding redundancy between users' actions (division of labor), either at the document level (Foley and Smeaton, 2009) or the role level (Pickens et al., 2008; Shah et al., 2010); (2) favoring the information flow among users (sharing of knowledge), either implicitly by search inference (Foley and Smeaton, 2010) or explicitly by collaborative-based interfaces (Morris and Horvitz, 2007); and (3) informing users of other collaborators' actions (awareness) (Dourish and Bellotti, 1992). Supporting these three principles remains a challenge in CIR, often tackled with adapted interfaces, revisited IR techniques, or collaborative document ranking models (Joho et al., 2009).

In this article, we focus more specifically on the third aspect when dealing with CIR models. In previous work, the focus has been on the mediation 
of collaborators' actions and complementarity skills in order to enhance the synergic effect within collaboration towards the satisfaction of the shared information need (Shah and González-Ibáñez, 2011b). Henceforth, division of labor is a pivotal issue for coordinating collaborators in terms of search actions with respect to their complementary skills. Assigning roles is one way of tackling this challenge because roles give a structure to the search process (Kelly and Payne, 2013). Beyond simply considering collaborators as peers and focusing on inferring the global relevance of documents towards all collaborators (Foley and Smeaton, 2009) or personalizing document scores for each collaborator (Morris et al., 2008), several works (Pickens et al., 2008; Shah et al., 2010; Soulier et al., 2013) propose assigning asymmetric roles to users in order to optimize the collaborative search effectiveness. Golovchinsky et al. (2009) suggested these roles in a role taxonomy.

Pickens et al. (2008) proposed a pair of roles, namely Prospector-Miner, that involved splitting a search task between the collaborators. The Prospector was responsible for formulating a search request that ensured search diversity, whereas the Miner was devoted to identifying highly relevant documents. Similarly, Shah et al. (2010) proposed a CIR model relying on the Gatherer-Surveyor relationship, in which the former's goal was to quickly scan search results and the latter focused on diversity. In these models, users' roles ensured a task-based division of labor.

Different from these works, Soulier et al. (2013) ensured the division of labor among collaborators by considering their respective domain expertise as the core source of a collaborator's role when aiming to solve a multi-faceted information need. For this purpose, the authors structured collaborators' actions by assigning documents to the most likely suited users, as well as allowing users to simultaneously explore distinct document subsets.

A new role-based approach has been proposed in (Soulier et al., 2014) which considers that collaborators' search behaviors were dynamic and that their role might evolve throughout the search session. This statement has been also outlined in (Tamine and Soulier, 2015). With this in mind, collaborators' predefined and labeled roles, namely Prospector-Miner and Gatherer/Surveyor, were identified in real time assuming a task-based division of labor policy based on their search behavior oppositions. Then, documents are ranked according to the CIR models associated with the mined roles (Pickens et al., 2008; Shah et al., 2010). 


\subsection{User Behavior Models for Document Retrieval}

The user behavior modeling domain focuses on the understanding of the user model within the search session. On one hand, some work (Evans and Chi, 2010; Yue et al., 2014) only focuses on the user modeling in a highly abstract level in order to build generative behavioral models. For instance, Yue et al. (2014) analyze temporal sequential data of collaborative search through a hidden Markov model. On the other hand, other research attempts to model user behaviors and to re-inject them into a retrieval model in order to enhance the search effectiveness (Agichtein et al., 2006). In the latter research domain, which is closer to our contribution, we distinguish three main lines of work based on feature-based document relevance prediction models (Agichtein et al., 2006; Radinsky et al., 2013), personalization approaches through users' preferences (Bennett et al., 2012; Teevan et al., 2005), or role extraction-based ranking models (Henderson et al., 2012; McCallum et al., 2007).

The first category of research that deals with prediction models analyzes several dimensions of user behaviors. In most of these works, a simplistic approach is usually followed that consists of integrating clickthrough data within the document scoring (Joachims, 2002) since this source of evidence expresses users' search behaviors. In addition, some authors (Agichtein et al., 2006) suggest a further abstraction level by proposing a robust user behavior model which takes the collective behaviors for reducing noise within an individual search session into account. Instead of smoothing individual behaviors with collective search logs, Radinsky et al. (2013) proposed another dimension of analysis that refines individual search logs through a temporal aspect that predicts queries and click frequencies within search behaviors. This user model relied on time-series and dynamically extracted topical trends re-injected within the ranking or the query auto-suggestion.

Beyond analyzing search behaviors for the purpose of document ranking, another line of work (Heath and White, 2008; White and Dumais, 2009) exploits search behaviors for predicting search engine switching events. These works' findings may be used to enhance the retrieval effectiveness and coverage of an information need, thus discouraging switching activities. For instance, dealing with the personalization approach, user profiles might be extracted considering users' relevance feedback (Bennett et al., 2012; Leung et al., 2008; Soulier et al., 2013; Teevan et al., 2005). In an individual search 
setting, Bennett et al. (2012) proposed to combine short-term and long-term search behaviors to mine users' interests. They built a multi-feature profile based on search history, query characteristics, document topic, and users' search actions. In contrast, Leung et al. (2008) modeled users' profiles through a concept-based representation inferred from clickthrough data. Each profile is then used to learn users' preferences with an SVM algorithm that personalizes their search results. Search personalization is also proposed in collaborative search settings (Morris et al., 2008; Soulier et al., 2013). For instance, Morris et al. (2008) integrated a personalized score (Teevan et al., 2005) within (1) a document smart-splitting over collaborators' rankings for retrieving individual rankings; and (2) a relevance summation of relevance feedback for building the final document list leveraging the collective relevance.

In the last category, previous work has proposed to model and/or mine users' roles from their search behaviors. In this context, contributions aim at either statistically identifying predefined roles (Golder and Donath, 2004; Kwak et al., 2010) or mining latent roles through probabilistic models (Henderson et al., 2012; McCallum et al., 2007). The first perspective relies on social network interactions for identifying labeled or predefined roles, such as "Celebrities or Ranters," through a statistical analysis, (Golder and Donath, 2004), or for identifying the "Network Leaders" using a PageRank-like algorithm (Pal and Counts, 2011) or a clustering method (Kwak et al., 2010). The second perspective offers a formal way to identify latent roles through the analysis of user interactions' similarities and dissimilarities (Henderson et al., 2012; McCallum et al., 2007). For instance, Henderson et al. (2012) focused on the transformation of a feature-based multidimensional matrix to identify the users' behavior model while McCallum et al. (2007) revised the LDA algorithm within a communication social network to mine the evolving roles of users according to message contents.

\subsection{Research Objectives}

From the literature review, one can infer that the key challenge in CIR concerns the difficulty of ranking documents in order to satisfy both individual and mutual goals with respect to the shared information need. Therefore, this challenge assumes that users are different and guided by complementary skills or knowledge (Sonnenwald, 1996). One possible way to consider users' 
differences might be to assign different roles with respect to their skills. However, CIR models based on predefined roles (Pickens et al., 2008; Shah et al., 2010; Soulier et al., 2013) raise two main concerns (Soulier et al., 2014):

1. The role assignment assumes that users behave the same way throughout the session by assigning roles to users at the beginning of the search session.

2. A role might not be in accordance with a user's intrinsic skills, and more particularly might not consider the ways in which these skills are most effective.

One solution is to derive users' roles from the differences and similarities they demonstrate in their interactions in order to exploit these roles within the ranking. For this purpose, two main approaches can be traced, which, unlike works focusing on user behavior models that mainly deal with users' intrinsic values (Agichtein et al., 2006; Bennett et al., 2012; Leung et al., 2008), consider users relative to their peers in order to highlight how they are the most effective. The first approach operates on a pool of predefined roles, and consists of a dynamic role assignment monitored by supervised learning, which is based on features inferred from users' interactions in the search system (Soulier et al., 2014). Once predefined roles have been identified, the associated state-of-the-art CIR models are used to solve the query. However, one limitation that could be raised from this work is that the labeled roles are predefined regardless of the users, thus restricting the likelihood that the identified roles exactly match collaborators' search skills. Therefore, two main challenges could be raised: (1) would a user be assigned to the role that best aligns with his/her behavior, even if it is not in particular accordance with his/her skills?; and (2) what if users align with multiple roles?

The second approach, which we utilize in this article, dynamically characterizes latent roles with an unsupervised learning method, rather than classifying roles in a taxonomy. More particularly, in contrast to well-know CIR models (Pickens et al., 2008; Shah et al., 2010) and in accordance with the limitations raised by Soulier et al. (2014), we approach here the problem of predefined roles, and propose to dynamically mine the unlabeled roles of collaborators throughout the search session in an unsupervised manner, and subsequently adapt the collaborative document ranking. As shown in Figure 


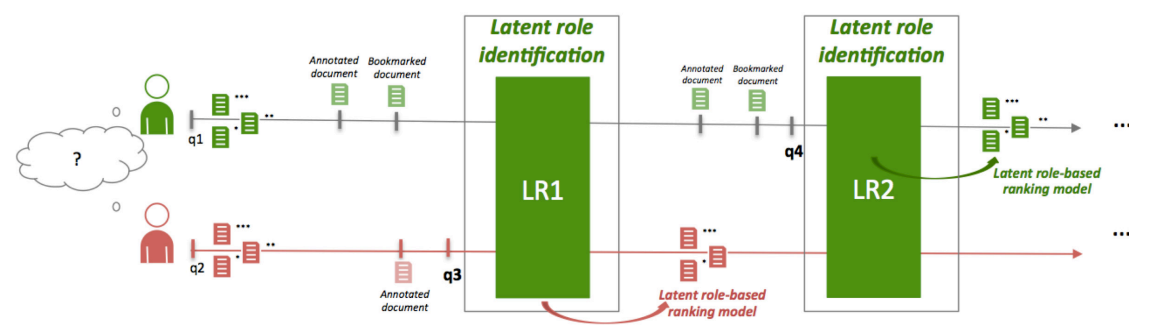

Figure 1: Unsupervised latent role learning methodology.

1, unlabeled roles of both collaborators are mined each time a user submits a query. Then, features modeling these unlabeled roles are re-injected in the collaborative document ranking model in order to display a ranked list of documents to this user.

In order to ensure our twofold objective of (1) mining unlabeled roles with respect to collaborators' behaviors and (2) collaboratively ranking documents, we rely on a feature set estimated at the document level. Therefore, we intuit that, if we consider a document's receipt of relevance feedback as a good indicator of users' search behaviors and preferences (Agichtein et al., 2006), those features assigned to the relevant document set would enable us to (1) mine latent roles of collaborators and (2) re-inject the mined latent roles within a CIR model.

Therefore, we aim to address the following research questions in this article:

- RQ1: How to infer collaborators' unlabeled roles through the differences and complementarities in their behaviors?

- RQ2: How to leverage these unlabeled roles for collaboratively ranking documents with respect to the shared information need?

We introduce the concept of "latent role," which captures collaborators' roles in real time according to the complementarity of their search skills, without any assumptions of predefined roles labeled or belonging to a role taxonomy (Golovchinsky et al., 2009). More particularly, guided by the division of labor policy, the users' latent roles leverage the skills in which collaborators are different-the most complementary and the most effective for 
enhancing the retrieval effectiveness of the search session. Also, we assume that collaborators' search skills might be inferred within the persistence of their search behaviors, since collaborators might have noisy search actions that could be due to the task, the topic, the interface design, or collaborators' engagement within the task. To this end, we are aware that this concept requires the search session to be synchronous, requiring the users to coordinate their actions and to exhibit their skills at the same time. Moreover, we assume that the users are constantly engaged in both generating/modifying the information needs as well as addressing them, and thus not being active, which could lead to noisy search actions or behaviors associated with inactivity.

\section{The model}

We present here our model based on the latent role of collaborators, assuming that users might refine their search strategies and behaviors throughout the session while they interact with their collaborators or assess search results.

Our model, called MineRank, considers search features modeling collaborators' behaviors and aims to rank documents in a collaborative manner at each query submission by leveraging collaborators' search skill complementarities. For convenience, we call an iteration associated to timestamp $t_{l}$, the time-window beginning at each time user $u$ submits query $q$ and ending while document list $\mathcal{D}_{u}^{t_{l}}$ is retrieved to user $u$. More particularly, an iteration of MineRank relies on two main steps illustrated in Figure 2: (1) learning across time the most discriminant feature set, which maximizes the differences between users' behaviors in search results in order to dynamically mine the latent role of collaborators (section 3.2), and (2) re-injecting latent roles for collaboratively ranking documents. For this purpose, we aim at predicting, through a learning model, the document relevance towards collaborators by taking into account their latent roles (section 3.3).

\subsection{Notations}

We consider a synchronous collaborative search session $S$ involving a pair of users $u_{1}$ and $u_{2}$ for solving a shared information need $I$ during a time interval $T$. Each user $u$ browses separately and formulates his/her queries for accessing their respective document result sets. As shown in Figure 1, 


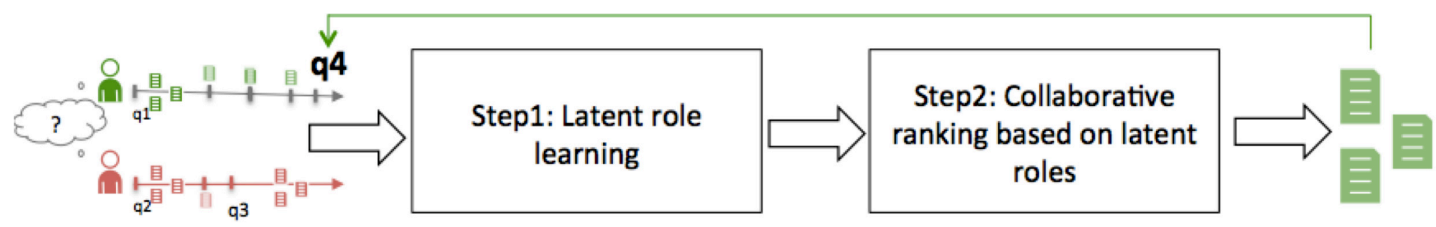

Figure 2: Minerank methodology for an iteration.

users have the possibility to perform different actions throughout the search session. Beyond formulating a query, they interact with the retrieved documents by visiting their content, annotating web pages with comments, bookmarking documents, or snipping pieces of information. Therefore, users' actions might be characterized by search behavior-based features, noted $F=\left\{f_{1}, \ldots, f_{k}, \ldots, f_{n}\right\}$. The latter expresses the set of $n$ features captured during the search session, detailed in Table 1. These features, based on the literature (Agichtein et al., 2006), fall under two categories:

- Submitted query features that capture collaborators' search experience with respect to the query topic. For instance, we integrate features based on the overlap between the query and pieces of a document (title, content, annotations/snippets generated by a user).

- Selected page features that capture collaborators' browsing behaviors in the search session in order to highlight time spent on webpages/on a specific domain as well as the specificity or readability of documents visited/annotated/snipped, and bookmarked by a given user.

We highlight that the feature set is slightly different from the one used in (Soulier et al., 2014) since the intuition of this proposed model is to re-inject the behavioral features within the collaborative document ranking. Furthermore, the feature set can be extended with no impact on the model.

Following Soulier et al. (2014), we represent a temporal feature-based user's behavior matrix $S_{u}^{\left(t_{l}\right)} \in \mathcal{R}^{t_{l} \times n}$, where $t_{l}$ is the timestamp. Each element $S_{u}^{\left(t_{l}\right)}\left(t_{j}, f_{k}\right)$ represents the average value of feature $f_{k}$ for user $u$ aggregated over the set $\mathcal{D}\left(u_{j}\right)^{\left(t_{j}\right)}$ of documents visited/annotated/snipped/bookmarked during the time interval $\left[0 \ldots t_{j}\right]$. Assuming that users' search behaviors might be refined throughout the session, the temporal modeling enables the 
characterization of the overall behavior of the user at timestamp $t_{l}$ avoiding the bias induced by noisy search actions.

Table 1: Search behavior features

\begin{tabular}{|c|c|c|}
\hline & Feature & Description \\
\hline \multirow{5}{*}{ 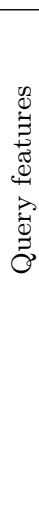 } & TitleOverlap (TiO) & $\begin{array}{l}\text { Fraction of shared words between query and } \\
\text { page title }\end{array}$ \\
\hline & TextOverlap (TeO) & $\begin{array}{l}\text { Fraction of shared words between query and } \\
\text { page content }\end{array}$ \\
\hline & AnnotationOverlap (AO) & $\begin{array}{l}\text { Fraction of shared words between query and } \\
\text { page annotation }\end{array}$ \\
\hline & SnippetOverlap (SO) & $\begin{array}{l}\text { Fraction of shared words between query and } \\
\text { snippet of the page }\end{array}$ \\
\hline & VisitedPosition (VP) & $\begin{array}{l}\text { Position of the URL in visited page order for } \\
\text { the query }\end{array}$ \\
\hline \multirow{6}{*}{ 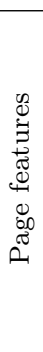 } & TimeQueryToPage (TQTP) & $\begin{array}{l}\text { Time between the query submission and the } \\
\text { visit of the page }\end{array}$ \\
\hline & TimeOnPage (TOP) & Page dwell time \\
\hline & TimeOnDomain (TOD) & Cumulative time for this domain \\
\hline & Readability (Read) & Document content readability \\
\hline & Specificity (Spec) & Document content specificity \\
\hline & Rating (Ra) & Rate of this page \\
\hline
\end{tabular}

According to Soulier et al. (2014), users' search skill difference toward a particular search feature $f_{k} \in F$ is referred to as $\Delta_{1,2}^{\left(t_{l}\right)}\left(f_{k}\right)$, where $\Delta_{1,2}^{\left(t_{l}\right)}\left(f_{k}\right)=S_{u_{1}}^{\left(t_{l}\right)}\left(f_{k}\right)-S_{u_{2}}^{\left(t_{l}\right)}\left(f_{k}\right)$. In addition, to ensure that both behaviors are found different in both users, and in order to identify those search behaviors that each user is better at than the other, we would like to know in which of those search behaviors each user is better than the other and highlight the acts in which he/she is the most effective with respect to his/her intrinsic skills as well as his/her collaborator's skills. With this in mind, correlations between collaborators' search feature differences were estimated, pair by pair, by adding the constraint that the difference between users for the implied features is significant, using the Kolmogorov-Smirnov test (p-value $p\left(\Delta_{1,2}^{\left(t_{l}\right)}\left(f_{k}\right)\right)$ ). Therefore, complementarities and similarities between collaborators $u_{1}$ and $u_{2}$ with respect to their search behaviors are emphasized through a correlation matrix $C_{1,2}^{\left(t_{l}\right)} \in \mathcal{R}^{p \times p}$ in which each element $C_{1,2}^{\left(t_{l}\right)}\left(f_{k}, f_{k^{\prime}}\right)$ is estimated as 
(Soulier et al., 2014):

$C_{1,2}^{\left(t_{l}\right)}\left(f_{k}, f_{k^{\prime}}\right)= \begin{cases}\rho\left(\Delta_{1,2}^{\left(t_{l}\right)}\left(f_{k}\right), \Delta_{1,2}^{\left(t_{l}\right)}\left(f_{k^{\prime}}\right)\right) & \text { if } p\left(\Delta_{1,2}^{\left(t_{l}\right)}\left(f_{k}\right)\right)<\theta \text { and } p\left(\Delta_{1,2}^{\left(t_{l}\right)}\left(f_{k^{\prime}}\right)\right)<\theta \\ 0 & \text { otherwise }\end{cases}$

As the goal is to focus on search behavior complementarities between unlabeled roles of collaborators $u_{1}$ and $u_{2}$, we assume that two features $f_{k}$ and $f_{k^{\prime}}$ behave similarly if the correlation $\rho\left(\Delta_{1,2}^{\left(t_{l}\right)}\left(f_{k}\right), \Delta_{1,2}^{\left(t_{l}\right)}\left(f_{k^{\prime}}\right)\right)$ of their difference is close to 1 . The closer to -1 the correlation is, the more complementary are collaborators' skills towards features $f_{k}$ and $f_{k^{\prime}}$. Focusing on users' difference $\Delta_{1,2}^{\left(t_{l}\right)}\left(f_{k}\right)$ towards search feature $f_{k}$ is not enough, since it does not ensure that collaborators' roles are complementary with respect to two search features: one user could be better for both features (Soulier et al., 2014), and, in this case, there is no need to leverage the other collaborator as a division of labor actor.

With this in mind, we introduce the concept of latent role based on the following hypothesis:

- H1: A latent role models the most significant similarities and complementarities between collaborators with respect to their search behaviors or skills throughout the search session in order to identify skills in which collaborators are the most effective.

- H2: Complementarities and similarities are respectively expressed by negative and positive correlations between search behavior features.

Therefore, at each timestamp, the latent role $L R_{1,2}^{\left(t_{l}\right)}$ highlights, for a pair of collaborators $u_{1}$ and $u_{2}$, their search skill differences and complementarities during the time period [0..t $t_{l}$; where search skills of a user are inferred from his/her temporal feature-based behavior matrix $S_{u}^{\left(t_{l}\right)}$ to highlight the persistence of unlabeled roles with respect to the task, the topic, the interface design or users' engagement within the task. Accordingly, the latent role $L R_{1,2}^{\left(t_{l}\right)}$ involves:

- A kernel $\mathcal{K}_{1,2}^{\left(t_{l}\right)}$ of a subset $F_{k 1,2}^{\left(t_{l}\right)} \subset F$ of $p$ behavioral features $F$, where $p$ is automatically defined by the latent role mining algorithm (see Section 3.2). In other words, $p$ expresses the number of the most significant 
features used to characterize the latent role according to hypothesis H1 and $\mathrm{H} 2$.

- A correlation matrix $C_{1,2}^{\left(t_{l}\right)} \in \mathcal{R}^{p \times p}$ that emphasizes complementarities and similarities between unlabeled roles of collaborators $u_{1}$ and $u_{2}$ with respect to their search behaviors.

\subsection{Learning Users' Latent Roles in Collaborative Search}

The underlying issue of the latent role mining consists of identifying the most discriminant features for characterizing collaborators' search behaviors which maximize, for a pair of collaborators, their complementarity. This leads us to propose a collaboration-oriented latent role mining approach based on a feature selection. The intuition behind our contribution is illustrated in Figure 3. The feature selection operates on the analysis of users' search behaviors. Once users have been identified as behaving differently towards search features, their complementary behaviors are modeled through a weighted network in order to identify the most important and discriminant features for characterizing collaborators' latent roles over the search session.

In what follows, we first express the optimization problem framework and the underlying assumptions. Then, we propose a method to solve the problem.

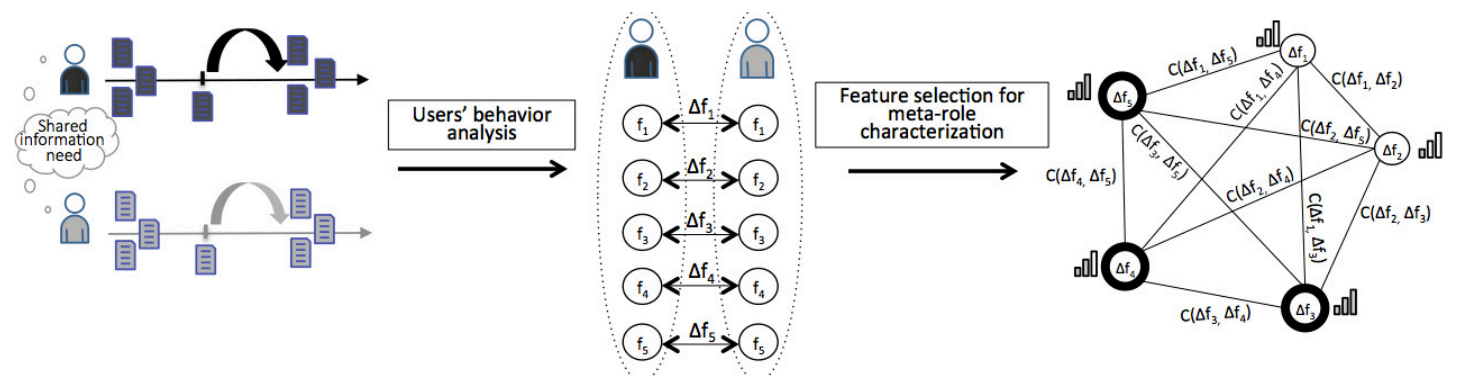

Figure 3: Unsupervised latent role learning methodology. 


\subsubsection{Latent Role Design}

Inspired from work proposed by Geng et al. (2007) and adapted to our collaborative latent role mining, the feature selection consists of building the latent role kernel $\mathcal{K}_{1,2}^{\left(t_{l}\right)}$ by identifying the smallest subset $F_{k 1,2}^{\left(t_{l}\right)}$ of $p$ features ( $p$ is undefined) according to three assumptions:

- A1: the importance $\mathcal{R} e c_{1,2}^{\left(t_{l}\right)}\left(f_{k}\right)$ of features $f_{k} \in F_{k 1,2}^{\left(t_{l}\right)}$ is dependent on their abilities to provide good indicators of the document assignment to users within the collaborative document ranking. We assume that a CIR model might support the division of labor, and that a document might be assigned to the most likely suited collaborator. As proposed by Shah et al. (2010), we formalize this principle through a document classification relying on relevance feedback collected throughout the search session until timestamp $t_{l}$, where each document cluster represents documents allocated to one of the collaborators. With this goal, we propose to cluster, using a 2-means classification, the set $\mathcal{D}^{\left(t_{l}\right)}$ of selected documents (through annotations/snippets/bookmarks) by both collaborators until timestamp $t_{l}$, according to the value of feature $f_{k}$. The cluster with the highest centroid is assigned to the collaborator $u_{j}$ with the highest value $S_{u_{1}}^{\left(t_{l}\right)}\left(f_{k}\right)$ whereas the other cluster is assigned to the other collaborator. We measure the quality of the classification based on feature $f_{k}$ towards each collaborator $u_{1}$ and $u_{2}$ using the recall measure $\mathcal{R} e c_{1,2}^{\left(t_{l}\right)}\left(f_{k}\right)$ :

$$
\mathcal{R e c}_{1,2}^{\left(t_{l}\right)}\left(f_{k}\right)=\frac{T P_{f_{k}}^{\left(t_{l}\right)}}{T P_{f_{k}}^{\left(t_{l}\right)}+F N_{f_{k}}^{\left(t_{l}\right)}}
$$

where $T P_{f_{k}}^{\left(t_{l}\right)}$ is the number of documents assigned to the cluster associated to the user who selected those documents using the 2-means classification based on feature $f_{k}$. For instance, if $u_{1}$ selected document $d_{1}$ before timestamp $t_{l}$, we consider a "True Positive" action if the classification algorithm attributes document $d_{1}$ to user $u_{1}$. Accordingly, $T P_{f_{k}}^{\left(t_{l}\right)}$ is incremented by 1 . Inversely, $F N_{f_{k}}^{\left(t_{l}\right)}$ expresses the number of documents not assigned to the cluster associated with the user who selected those documents. For instance, if document $d_{1}$ is attributed to cluster of user $u_{2}, F N_{f_{k}}^{\left(t_{l}\right)}$ is incremented of 1 .

- A2: the redundancy between features might be avoided in order to consider only the most discriminant ones for characterizing latent roles 
through complementary search behaviors among users, modeled using feature correlations $C_{1,2}^{\left(t_{l}\right)}\left(f_{k}, f_{k^{\prime}}\right)$. We investigate here how to identify the most discriminant features for characterizing users' roles, and more particularly features highlighting complementary search behaviors among users. The main assumption is to identify for which skills collaborators are the most suited with respect to their co-collaborators for solving the shared information need. We used the correlation $C_{1,2}^{\left(t_{l}\right)}\left(f_{k}, f_{k^{\prime}}\right)$ between collaborators' differences $\Delta_{1,2}^{\left(t_{l}\right)}\left(f_{k}\right)$ and $\Delta_{1,2}^{\left(t_{l}\right)}\left(f_{k^{\prime}}\right)$ towards a pair of search behavior features $f_{k}$ and $f_{k^{\prime}}$.

- A3: the feature selection must maximize the importance $\mathcal{R} e c_{1,2}^{\left(t_{l}\right)}\left(f_{k}\right)$ of the selected features $f_{k} \in F_{k 1,2}^{\left(t_{l}\right)}$ within the collaborative document ranking and minimize the redundancy $C_{1,2}^{\left(t_{l}\right)}\left(f_{k}, f_{k^{\prime}}\right)$ between the pairwise selected features. Thus, we formalize the feature selection algorithm as the following optimization problem:

$$
\begin{array}{rc}
\max _{\alpha} & \sum_{k=1}^{n} \mathcal{R e c}_{1,2}^{\left(t_{l}\right)}\left(f_{k}\right) \cdot \alpha_{k} \\
\min _{\alpha} & \sum_{k=1}^{n} \sum_{k^{\prime}=1}^{n} C_{1,2}^{\left(t_{l}\right)}\left(f_{k}, f_{k^{\prime}}\right) \cdot \alpha_{k} \cdot \alpha_{k^{\prime}} \\
\text { subject to } & \alpha_{k}=\{0,1\} ; k=1, \ldots, n \\
\text { and } & \sum_{k=1}^{n} \alpha_{k}=p
\end{array}
$$

where $\alpha$ is the vector of size $n$ where each element $\alpha_{k}$ is a Boolean indicator specifying whether feature $f_{k}$ is included in the feature subset $F_{k 1,2}^{\left(t_{l}\right)}$ at timestamp $t_{l}$.

This optimization problem with multi-objectives might be transformed as a unique objective optimization problem by linearly combining both optimization functions.

$$
\begin{array}{rc}
\max _{\alpha} \sum_{k=1}^{n} \mathcal{R e c}_{1,2}^{\left(t_{l}\right)}\left(f_{k}\right) & \cdot \alpha_{k}-\gamma\left(\sum_{k=1}^{n} \sum_{k^{\prime}=1}^{n} C_{1,2}^{\left(t_{l}\right)}\left(f_{k}, f_{k^{\prime}}\right) \cdot \alpha_{k} \cdot \alpha_{k^{\prime}}\right) \\
\text { subject to } & \alpha_{k}=\{0,1\} ; k=1, \ldots, n \\
\text { and } & \sum_{k=1}^{n} \alpha_{k}=p
\end{array}
$$

where $\gamma$ is a decay parameter expressing the level of behavior complementarity taken into account in the latent role mining algorithm. This 
parameter is fixed over the session since we hypothesize that the ratio between the feature importance and complementarity does not depend on the collaborators' current latent roles at timestamp $t_{l}$.

\subsubsection{Latent Role Optimization}

Our optimization problem defined in Equation 4 might be resolved by undertaking all the possible feature combinations of size $p$, where $p=2, \ldots, n$. Although optimal, this method is time-consuming with a complexity of up to $O\left(\sum_{p=1}^{n} C_{n}^{p}\right)$.

We propose, here, a graph-based resolution algorithm attempting to identify the best feature subset which may provide a locally optimal solution, but is more practically applicable with complexity which could reach a maximum of $O\left(3^{\frac{n}{3}}\right)$. The main objective is to extract the smallest feature node set which enhances the importance of the set of retained features while maximizing the differences of collaborators within their search behaviors. In this context, we represent features through a collaboration-based graph $G_{1,2}^{\left(t_{l}\right)}$ modeling search behaviors of collaborators $u_{1}$ and $u_{2}$ at timestamp $t_{l}$. The graph $G_{1,2}^{\left(t_{l}\right)}=\left(A_{1,2}^{\left(t_{l}\right)}, C_{1,2}^{\left(t_{l}\right)}\right)$, illustrated in Figure A.10, involves nodes $A_{1,2}^{\left(t_{l}\right)}$ which represent each feature $f_{k} \in F$, weighted by an importance measure $\mathcal{R} e c_{1,2}^{\left(t_{l}\right)}\left(f_{k}\right)$ within the collaborative document ranking, and undirected weighted edges $C_{1,2}^{\left(t_{l}\right)}: \mathcal{R}^{F \times F}$ which represent collaborators' search behavior similarities or complementarities by considering the correlation $C_{1,2}^{\left(t_{l}\right)}\left(f_{k}, f_{k^{\prime}}\right)$ between differences of pairwise features $f_{k}$ and $f_{k^{\prime}}$.

The used notations are detailed in Table 2. In what follows, we describe the algorithm, called Coll-Clique, for solving the optimization problem (Algorithms 1 and 2). Note that an illustration of our algorithm is presented in Appendix A.

Table 2: Notations

\begin{tabular}{ll}
\hline Notation & Description \\
\hline$C$ & The feature graph representing the growing clique \\
$P$ & The evolving candidate graph \\
$K$ & The maximum clique satisfying the optimization problem \\
$N b h d(C)$ & $\begin{array}{l}\text { The function that returns in a decreasing order the neighboring } \\
\text { features of all features belonging to C with non null weight }\end{array}$ \\
$N d s(K)$ & The function that returns all the features belonging to $K$ \\
\hline
\end{tabular}




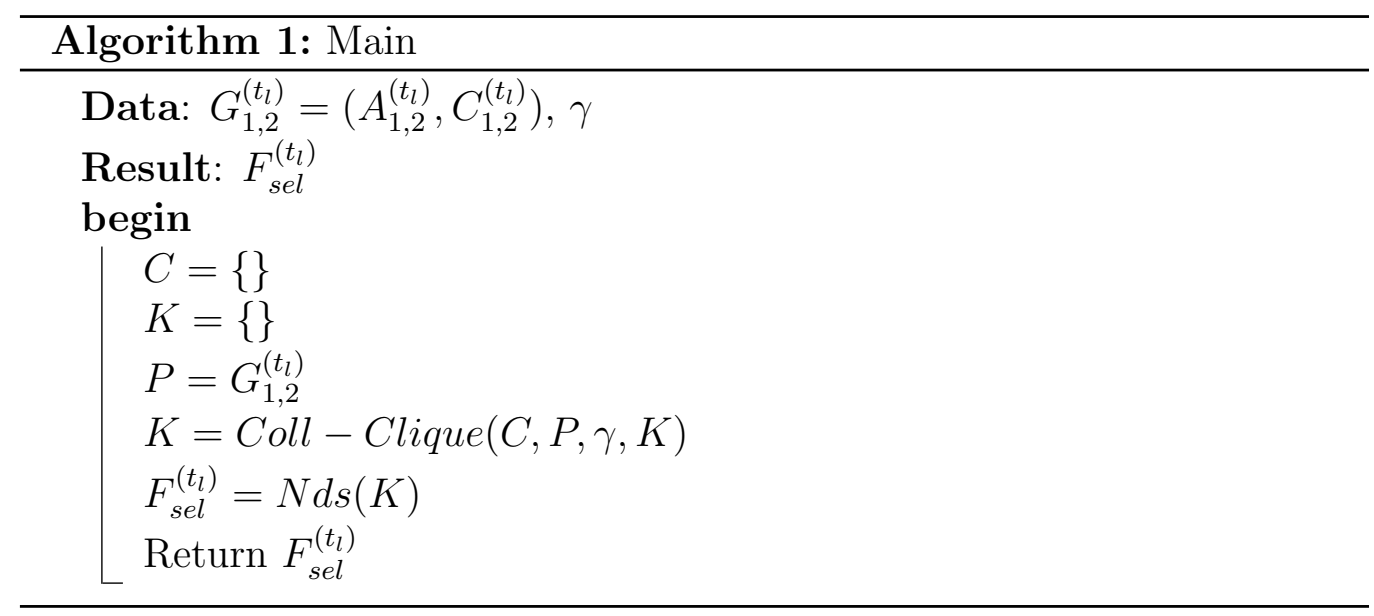

In order to solve the optimization problem, we extended the maximum clique algorithm (Carraghan and Pardalos, 1990) in order to fit with our feature selection problem in a collaborative context. Our intuition is that a weighted graph is complete since it models search behavior complementarities through correlations between pairwise features. The Coll-Clique algorithm, rather than focusing on a node level for identifying the biggest complete subgraph (Carraghan and Pardalos, 1990), also called the maximum clique, aims at extracting the subgraph which maximizes the node weights, namely the search feature importances (assumption A1), and minimizes the relationship weights between nodes, namely pairwise search behavior correlations for both collaborators (assumption A2).

As shown in Algorithm 1, Coll-Clique relies on two feature graphs:

- The growing clique $C$, candidate to be the maximum clique $K$.

- The feature graph $P$, which includes candidate features to be added to the growing clique $C$. Nodes in $P$ are obtained through the function $N d s(P)$.

Initially, $C$ is empty and $P$ is the graph including all the features. The algorithm, as shown in Algorithm 2, recursively increments the growing clique $C$ using features $f_{h}$ involved within graph $P$ built upon the function $\operatorname{Nbhd}(C)$, which creates a new candidate feature graph $P$ that only retrieves in a decreasing order features characterized by a positive depreciated weight. This operation is noted $C \oplus f_{h}$. At each recursion, the weight $\mathcal{R e c}_{1,2}^{\left(t_{l}\right)}\left(f_{k^{\prime}}\right)$ of 


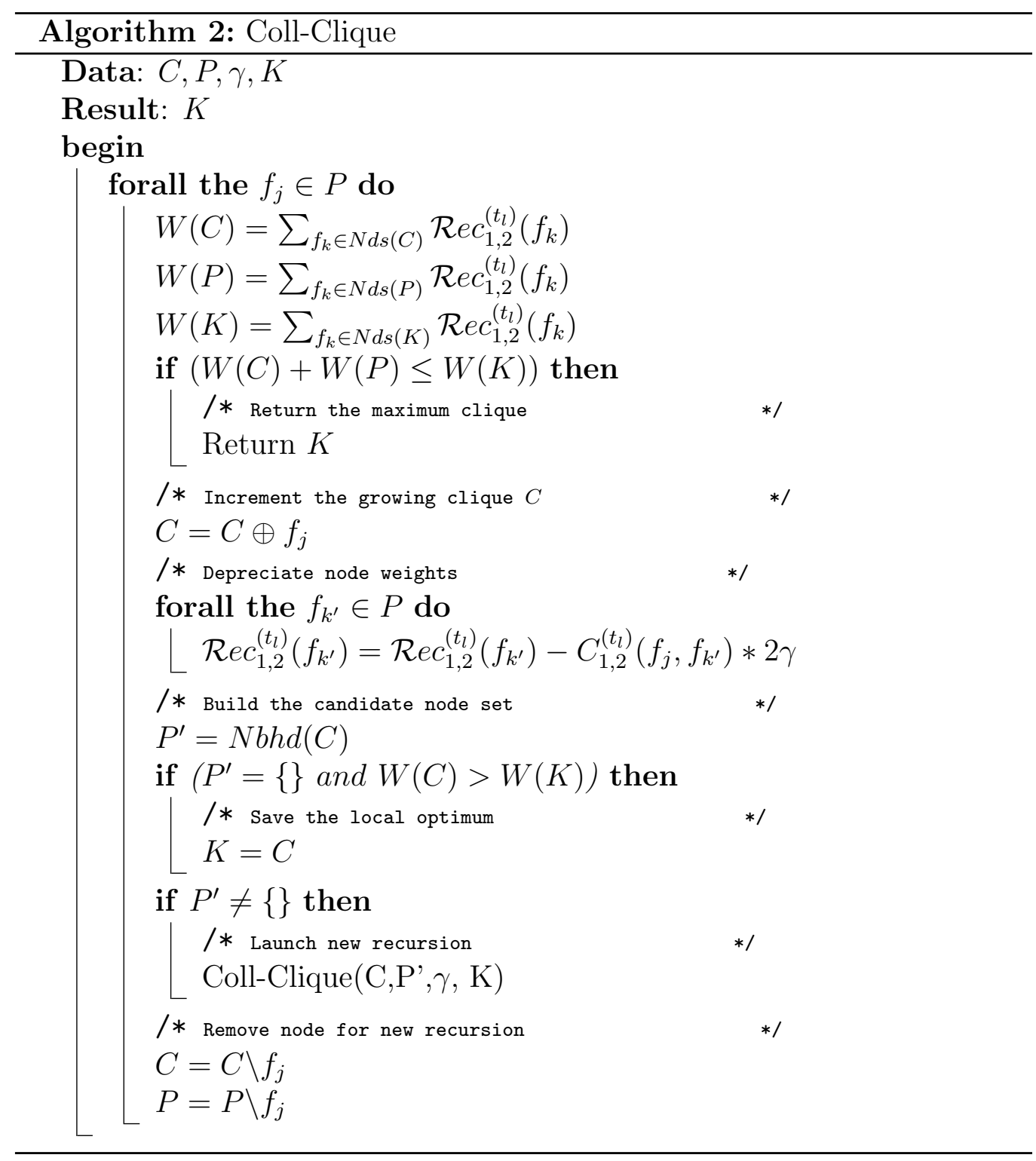

the other remaining features $f_{k^{\prime}}$ is depreciated by the correlation $C_{1,2}^{\left(t_{l}\right)}\left(f_{j}, f_{k^{\prime}}\right)$ with respect to the last selected feature $f_{j}$.

Let us denote $W(K)$ as the sum of feature weights within the maximum clique $K$. We assume that this sum refers to the indicator we would like to 
maximize (Equation 4) since the feature weight (importance $\mathcal{R} e c_{1,2}^{\left(t_{l}\right)}\left(f_{k^{\prime}}\right)$ ) is recursively depreciated with respect to the adjacent edge weight (correlation $\left.C_{1,2}^{\left(t_{l}\right)}\left(f_{j}, f_{k^{\prime}}\right)\right)$. If the weight $W(C)+W(P)$ of features within $C$ and $P$ is lower than the weight $W(K)$ within $K$ identified until the current iteration, there is no way to build a clique from $C$ by adding features from $P$ with a higher weight than the weight of features in $K$. Finally, the set of selected features $F_{k 1,2}^{\left(t_{l}\right)}$ of size $p$ inferred from the $p$ nodes within the maximum clique $K$ builds the latent role kernel $\mathcal{K}_{1,2}^{\left(t_{l}\right)}$.

We highlight that our algorithm strictly follows the framework of the maximum clique extraction algorithm proposed by Carraghan and Pardalos (1990), which is the initial version of the branch-and-bound algorithm category, well-known to ensure the guarantee of the optimal solution (Wu and Hao, 2015). We add one heuristic in order to consider a weighted graph aiming at solving an optimization problem, initially proposed by Geng et al. (2007) and adapted to our problem. Therefore, given that the candidate clique $C$ in incremented by positively-weighted nodes in a decreasing order, one could assume that the equation $(W(C)+W(P) \leq W(K))$ aims at maximizing the weight of the maximum clique $K$ where its weight could be estimated as follows:

$$
W(K)=\sum_{k=1}^{|K|} \mathcal{R} e c_{1,2}^{\left(t_{l}\right)}\left(f_{k}\right)-\gamma\left(\sum_{k=1}^{|K|} \sum_{k^{\prime} \neq k ; k^{\prime}=1}^{|K|} C_{1,2}^{\left(t_{l}\right)}\left(f_{k}, f_{k^{\prime}}\right)\right)
$$

The first part of the equation refers to the initial weight of nodes $f_{k}$ in the initial graph $\mathcal{G}$ while the second part expresses the depreciation of the node weight with respect to the nodes belonging to the maximum clique. Therefore, maximizing the weight of the maximum clique $K$ is equivalent to solving the optimization problem presented in Equation 4.

\subsection{Latent Role-based Collaborative Document Ranking}

In this section, we re-inject the latent role kernel identified in the previous section in order to collaboratively rank documents to users. The idea is to use the most discriminant features-characterizing search behavior complementarities of both collaborators-to first assign documents to the most likely suited collaborator, in order to ensure the division of labor, and then rank 


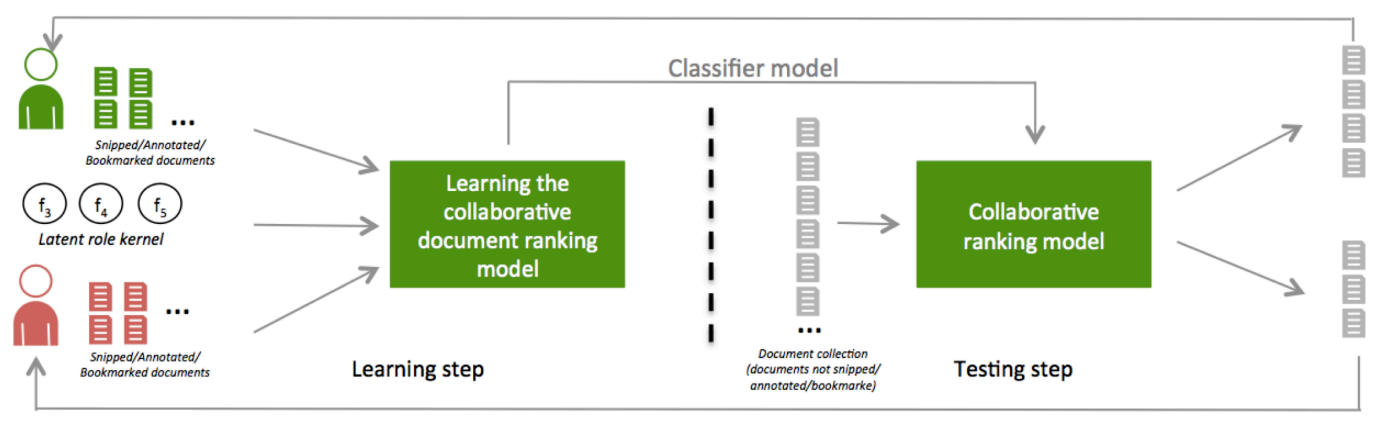

Figure 4: Overview of the collaborative document ranking using latent role of collaborators

the documents assigned to each user. For this purpose, we use a classifier learning algorithm which operates on the document representation restricted to features implied within the latent role $M_{1,2}^{\left(t_{l}\right)} \in \mathcal{R}^{m \times m}$ at timestamp $t_{l}$. We highlight that only the document list $\mathcal{D}_{u}^{t_{l}}$ associated with the class of the user $u$ who submitted query $q$ is displayed. Indeed, as explained in Figure 1, we only attempt to satisfy the information need of the user who submitted the query. We assume that the other collaborator $u^{\prime}$ might not be interested in query $q$ and is already examining a document list $\mathcal{D}_{u^{\prime}}^{t_{l}^{\prime}}$ retrieved with respect to a previously submitted query at timestamp $t_{l}^{\prime}<t_{l}$. We choose to use the Logistic Regression as the classifier learning algorithm which, as usual, runs into two stages illustrated in Figure 4:

- Stage 1. The learning step considers the set $\mathcal{D}^{\left(t_{l}\right)}$ of snipped/bookmarked/annotated documents by either collaborator $u_{1}$ or $u_{2}$ before timestamp $t_{l}$. Documents selected by both collaborators are removed from this set, since they are not discriminant for the collaborative-based document allocation to collaborators. Each document $d_{i} \in \mathcal{D}^{\left(t_{l}\right)}$ is modeled by a feature vector $x_{i}^{\left(t_{l}\right)} \in \mathcal{R}^{m}$, estimated according to value of the feature $f_{k} \in \mathcal{K}_{1,2}^{\left(t_{l}\right)}$ for document $d_{i}$ with respect to collaborators' actions and timestamp $t_{h}$ of its assessment, with $t_{h} \leq t_{l}$. Document $d_{i}$ also receives a classification variable $c_{i}^{\left(t_{l}\right)} \in\{0 ; 1\}$ where values 0 and 1 express the class of collaborators, respectively $u_{1}$ and $u_{2}$, who have selected this document.

The objective of the document ranking learning function is to iden- 
tify the predictor weight vector $\beta_{j}^{\left(t_{l}\right)} \in \mathcal{R}^{m}$ in order to estimate the probability of allocating documents to collaborator $u_{j} \in\left\{u_{1}, u_{2}\right\}$. The logistic regression aims at maximizing the likelihood $l$ detailed in Equation 6 which relies on the logit function formalized in Equation 7 . The latter models the probability $P_{j}\left(x_{i}^{\left(t_{l}\right)}\right)$ for document $d_{i}$ belonging to user class $c_{i} \in\{0 ; 1\}$ with respect to feature vector $x_{i}^{\left(t_{l}\right)}$.

$$
\begin{array}{rc}
\max _{\beta_{j}^{\left(t_{l}\right)}, \beta_{j}^{\left(t_{l}\right)}} \quad \sum_{d_{i} \in \mathcal{D}^{\left(t_{l}\right)}}\left(c_{i} \cdot \ln \left(P_{j}\left(x_{i}^{\left(t_{l}\right)}\right)\right)\right) \\
\\
\text { where } P_{j}\left(x_{i}^{\left(t_{l}\right)}\right)=\frac{}{+\left(1-c_{i}\right) \ln \left(1-P_{j}\left(x_{i}^{\left(t_{l}\right)}\right)\right)}
\end{array}
$$

- Stage 2. The testing step considers the set $\mathcal{D}_{n s e l}^{\left(t_{l}\right)}$ of documents not selected by both collaborators $u_{1}$ and $u_{2}$ before timestamp $t_{l}$. The feature vector $x_{i}^{\left(t_{l}\right)}$ is estimated according to feature average values of document $d_{i}$ with respect to the search logs collected before timestamp $t_{l}$, not necessarily by both collaborators. Indeed, there is no available value for action-based features, such as AnnotationOverlap, for the pair of collaborators considering that the document has not been collected by the pair of users. The fitted model learnt through the logistic regression algorithm estimates the probability $P_{j}\left(x_{i}^{\left(t_{l}\right)}\right)$ of assigning document $d_{i^{\prime}} \in \mathcal{D}_{n s e l}^{\left(t_{l}\right)}$ to the collaborator class $c_{i}$ with respect to the predictor weight $\beta_{j}^{\left(t_{l}\right)}$. Document $d_{i^{\prime}}$ is allocated to the collaborator class $c_{j}$ with the highest probability $P_{j}\left(x_{i}^{\left(t_{l}\right)}\right) ; \forall j \in\{0,1\}$, which is also used for ranking documents within the collaborator class.

Moreover, we add a supplementary layer of division of labor by ensuring that result lists $\mathcal{D}_{u^{\prime}}^{t_{l}}$ and $\mathcal{D}_{u^{\prime}}^{t_{l}^{\prime}}$ simultaneously displayed (even if retrieved at different timestamps $t_{l}$ and $t_{l}^{\prime}$ ) to collaborators $u$ and $u^{\prime}$ include distinct documents.

\section{Experimental Evaluation}

We performed an experimental evaluation investigating the impact of mining latent roles of collaborators on the retrieval effectiveness of a collaborative document ranking model. The following are the hypotheses that guide our investigation: 
1. A CIR model should fit with collaborators' complementary skills in which they are the most effective with respect to the collaborative setting, taking into account their whole behaviors regardless of their skills or predefined roles.

2. A CIR model should achieve a greater effectiveness than users working separately.

3. A CIR model should dynamically assign collaborators' roles in an unsupervised manner in the search session instead of assigning roles regardless of their skills.

In what follows, we describe the experimental protocol and present the obtained results.

\subsection{Protocol Design}

\subsubsection{User studies}

Since no well-established benchmark exists in the CIR domain, we used search logs collected from two different collaborative-based user studies, US1 and US2, supported by a collaborative search system (Shah and GonzálezIbáñez, 2011a) based on user mediation. The system allows users to browse the web and submit queries on independent search engines, mainly Google. The system includes a toolbar and a sidebar, providing a functionality in which users can interact with their peers through an instant messaging system as well as bookmarking, annotating and snipping web pages. Moreover, the sidebar ensures awareness by showing a user what he/she, as well as his/her collaborator, have bookmarked/snipped/annotated during the session. In addition, the system tracks collaborators' activities and records their search logs-such as visited pages, submitted queries, and relevance feedback-all over the session. An overview of the used system is illustrated in Figure 5. We outline that this system ensures the awareness paradigm since the sidebar allows collaborators to be aware of other relevance feedback.

The user studies, US1 and US2, involved respectively 25 non-native and 10 native English user pairs (a total of 70 people) who were recruited from university campuses and received compensation for their involvement within the experiments ( $\$ 20$ per person, with an additional $\$ 50$ for the three best performing groups). Accordingly, these participants performed the task of an exploratory search problem within 30 minutes in a co-located setup in 


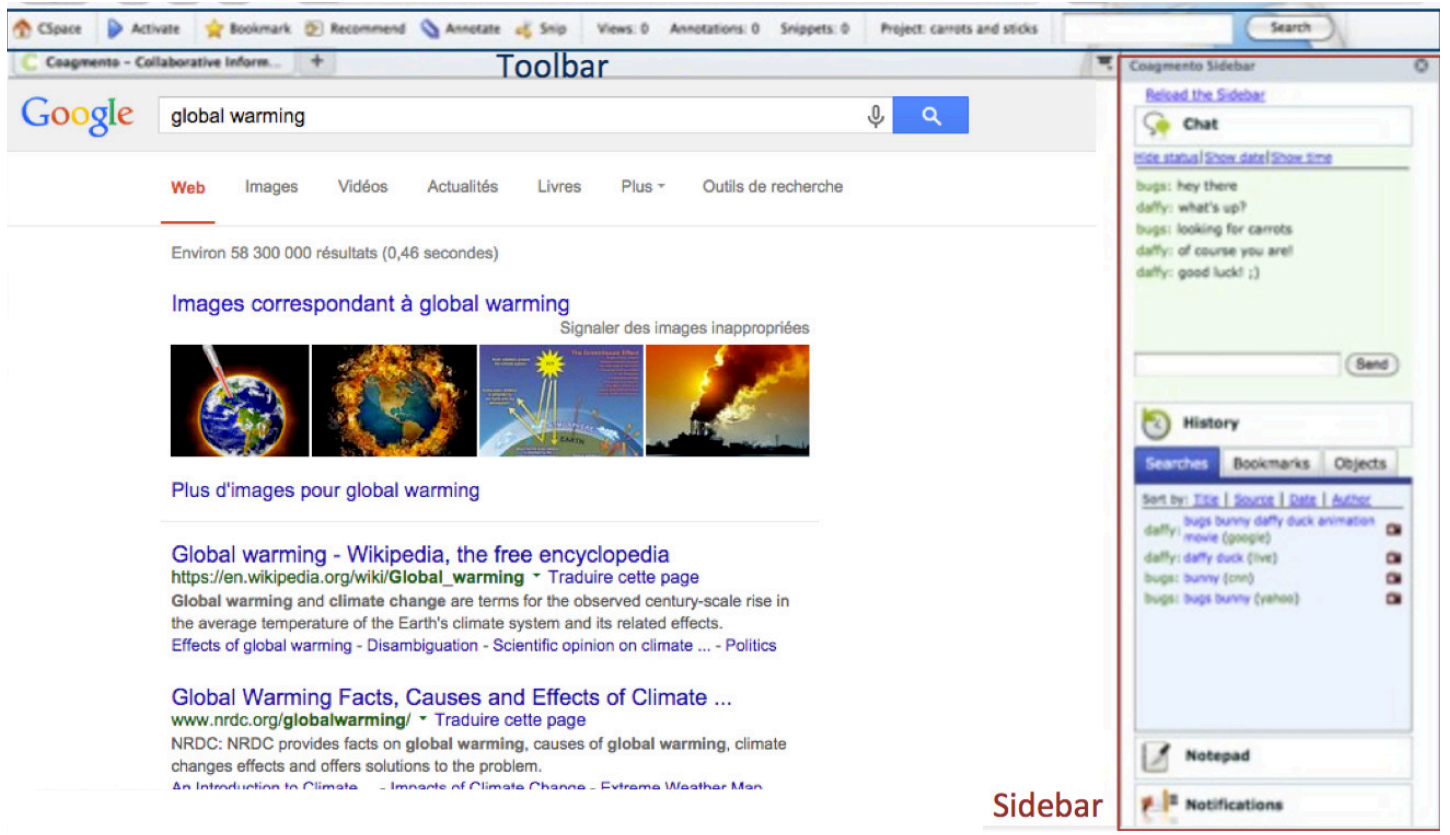

Figure 5: Coagmento system

their mother tongue. During the task, the collaborators interacted with each other in order to identify as many relevant documents as possible. Their interactive behaviors generally involved discussions about their search strategies or linked sharing exchange, always through the chat system. For user study US2, participants also had to write a report using web pages saved throughout the search session, which allowed them less time to browse on the web. Task topics are, respectively, "tropical storms" and "global warming". Guidelines of these two tasks are expressed in Table 3.

Statistics of both user studies are shown in Table 4. An analysis of participants' submitted queries shows that they are mainly a reformulation of topics, as each topical word often occurs in the queries. Indeed, among the 1174 submitted queries for US1, the terms "tropical" and "storm" are used 1077 and 1023 times, respectively, whereas the terms "global" and "warming" are used 254 and 247 times over the 313 submitted queries in US2. During the task, participants examined, respectively, 91 and 73 webpages for US1 and US2. We highlight that the number of submitted queries and visited 
Table 3: Collaborative tasks in user studies US1 and US2

\begin{tabular}{|c|c|}
\hline Tasks & tuidelines \\
\hline US1 & $\begin{array}{l}\text { The mayor of your countryside village must choose between building a } \\
\text { huge industrial complex or developing a nature reserve for animal con- } \\
\text { servation. As forest preservationists, you must raise awareness about the } \\
\text { possibility of wildlife extinction surrounding such an industrial complex. } \\
\text { Yet, before warning all citizens, including the mayor, you must do ex- } \\
\text { tensive research and collect all the facts about the matter. Your objective } \\
\text { is to collaboratively create a claim report, outlining all the possible out- } \\
\text { comes for wildlife should the industrial complex be built. Your focus is } \\
\text { on wildlife extinction. You must investigate the animal species involved, } \\
\text { the efforts done by other countries and the association worldwide to pro- } \\
\text { tect them and the reasons we, as humans, must protect our environment } \\
\text { in order to survive. You must identify all relevant documents, facts, and } \\
\text { pieces of information by using bookmarks, annotations, or saving snip- } \\
\text { pets. If one document discusses several pieces of useful information, you } \\
\text { must save each piece separately using snippets. Please assume that this } \\
\text { research task is preliminary to your writing, enabling you to provide all } \\
\text { relevant information to support your claims in your report. }\end{array}$ \\
\hline US2 & $\begin{array}{l}\text { A leading newspaper agency has hired your team to create a compre- } \\
\text { hensive report on the causes, effects, and consequences of the climate } \\
\text { change taking place due to global warming. As a part of your contract, } \\
\text { you are required to collect all the relevant information from any avail- } \\
\text { able online sources that you can find. To prepare this report, search and } \\
\text { visit any website that you want and look for specific aspects as given in } \\
\text { the guideline below. As you find useful information, highlight and save } \\
\text { relevant snippets. Later, you can use these snippets to compile your } \\
\text { report, no longer than 200 lines. Your report on this topic should ad- } \\
\text { dress the following: Description of global warming, scientific evidence of } \\
\text { global warming affecting climate change, causes of global warming, con- } \\
\text { sequences of global warming causing climate change, and measures that } \\
\text { different countries around the globe have taken over the years to address } \\
\text { this issue, including recent advancements. Also describe different view- } \\
\text { points people have about global warming (specify at least three different } \\
\text { viewpoints you find) and relate those to the the aspects of controversies } \\
\text { surrounding this topic. }\end{array}$ \\
\hline
\end{tabular}


pages by collaborative groups are higher for US1; this could be explained by the additional objective of participants in US2, which consisted of writing a report. The latter left participants less time for browsing the web.

\begin{tabular}{lcc}
\hline & US1 & US2 \\
\hline Topic & Tropical storm & Global warming \\
Number of dyads & 25 & 10 \\
Total number of visited pages & 4734 & 1935 \\
Total number of bookmarked/rated pages & 333 & - \\
Total number of snipped pages & 306 & 208 \\
\hline Total number of submitted queries & 1174 & 313 \\
Average number of terms by query & 3.65 & 4.73 \\
\hline
\end{tabular}

Table 4: Statistics of user studies US1 and US2

\subsubsection{Data}

Given the distinct languages of both user studies, we build two separate document indexes. Respectively, for each user study US1 and US2, we aggregated the respective web pages seen by the whole participant set as well as the top 100 search engine result pages (SERPs) from Google for the submitted query set. We highlight that the SERPs were extracted later in order to avoid processing overload. Each web page was processed for extracting $<$ title $>$ and $<\mathrm{p}>$ tags. In order to increase the size of document indexes, we carried out this protocol for other proprietary user studies performed in other collaborative settings, not considered for our experiments (Shah and González-Ibáñez, 2011a). In the end, the indexes included 24, 226 and 74, 844 documents for US1 and US2, respectively.

\subsubsection{Evaluation protocol}

In order to avoid a bias that could be involved in a re-ranking approach that relies on features measuring the similarity of the document with respect to the query, we highlight that the collaborative ranking step is carried out on the whole document collection. With this in mind, we consider two versions of our model:

- MineRank(q): our proposed unsupervised ranking model in which the latent role mining and the collaborative ranking steps are successively launched at each query submission. 
- MineRank(t): our proposed unsupervised model in which the collaborators' latent role mining (section 3.2) is performed at regular timestamps $t$ similar to Soulier et al. (2014), while the collaborative ranking step (section 3.3) is launched at each query submission by considering the latent role mined at the last timestamp $t$.

We highlight that, similar to Soulier et al. (2014), the BM25 model is launched when no search skill differences between collaborators are detected.

For effectiveness comparison, we ran the following baselines at each query submission:

- BM25: the BM25 ranking model which refers to an individual setting. This setting simulates a search session in which collaborators perform their search task on independent search engines.

The BM25 ranking model (Robertson and Walker, 1994) estimates the similarity score between document $d_{i}$ and query $q_{h}$ as:

$$
R S V\left(d_{i}, q_{h}\right)=\sum_{t_{v} \in q_{h}} \frac{N-n_{v}+0.5}{n_{k}+0.5} \frac{f_{i v} \cdot\left(k_{1}+1\right)}{f_{i v}+k_{1} \cdot\left(1-b+b \cdot \frac{\left|d_{i}\right|}{a v g_{d l}}\right)}
$$

where $N$ expresses the collection size, $n_{v}$ the number of documents including term $t_{v}$. The frequency of term $t_{v}$ in document $d_{i}$ is noted $f_{i v} . \quad\left|d_{i}\right|$ represents the length of document $d_{i}$ whereas the average document length is noted $a v g_{d l}$. $k_{1}$ and $b$ are model parameters.

- Logit: the CIR model which only involves the last step of our algorithm (section 3.3) considering the whole set of features in order to measure the effectiveness of a personalized search without any consideration of the latent roles.

- PM: the CIR model refers to a system-based mediation guided by predefined fixed roles of Prospector-Miner (Pickens et al., 2008).

According to collaborators' roles, this model relies on two different ranking functions:

1. The query term suggestion function aims at favoring the Prospector's search diversity. For each term $t_{k}$ belonging to documents 
previously retrieved in lists $L$, its score is estimated as:

$$
\operatorname{score}\left(t_{k}\right)=\sum_{L_{h} \in L} w_{r}\left(L_{h}\right) w_{f}\left(L_{h}\right) r l f\left(t_{k}, L_{h}\right)
$$

where $r l f\left(t_{k}, L_{h}\right)$ expresses the number of documents with term $t_{k}$ in list $L_{h}$.

2. The document ranking function ensures the relevance of documents not examined by the Prospector towards the topic. The score of document $d_{i}$ is estimated as follows:

$$
\operatorname{score}\left(d_{i}\right)=\sum_{L_{h} \in L} w_{r}\left(L_{h}\right) w_{f}\left(L_{h}\right) b o r d a\left(d_{i}, L_{h}\right)
$$

where $\operatorname{bord} a\left(d_{i}, L_{h}\right)$ is a voting function.

These two functions are based on relevance and freshness factors estimated as follows:

- The relevance factor $w_{r}\left(L_{h}\right)$ which estimates the ratio of relevant documents in list $L_{h}$ retrieved for query $q_{h}$, noted $\mid$ rel $\in L_{h} \mid$ with the number of non-relevant documents in the same list, noted $\mid$ nonrel $\in L_{h} \mid:$

$$
w_{r}\left(L_{h}\right)=\frac{\mid \text { rel } \in L_{h} \mid}{\mid \text { nonrel } \in L_{h} \mid}
$$

- The freshness factor $w_{f}\left(L_{h}\right)$ which estimates the ratio of documents not visited in list $L_{h}$, noted $\mid$ nonvisit $\in L_{h} \mid$, with the number of visited documents in $L_{h}$, noted $\mid$ visit $\in L_{h} \mid$ :

$$
w_{f}\left(L_{h}\right)=\frac{\mid \text { nonvisit } \in L_{h} \mid}{\mid \text { visit } \in L_{h} \mid}
$$

- GS: the CIR model refers to a system-based mediation guided by predefined fixed roles of Gatherer-Surveyor (Shah et al., 2010).

This model is launched after query submissions $\left(q_{h}\right.$ and $\left.q_{h^{\prime}}\right)$ of each collaborator (respectively, $u_{j}$ and $u_{j^{\prime}}$ ) and consists of two steps:

1. The Merging step in which document lists are merged using the CombSUM function. 
2. The Splitting step in which documents in the merging list are classified using a 2-means algorithm. Each cluster is assigned to a collaborator using the following criteria: the cluster with the highest gravity center is assigned to the Gatherer whereas the remaining cluster is assigned to the Surveyor.

- RoleMining: the user-driven system-mediated CIR model which mines predefined roles of collaborators in real time and ranks documents according to the associated state of the art CIR models (Soulier et al., 2014). In contrast to our proposed approach, this setting considers roles (namely Gatherer/Surveyor and Prospector/Miner) predefined in a role taxonomy Golovchinsky et al. (2009) that might not exactly fit with users' skills.

This model exploits the correlation matrix denoting collaborators' behaviors in order to assign users predefined roles, modeled through a role pattern. In particular, according to a role pattern pool, the role-based identification assigns the role pattern correlation matrix $F^{R_{1,2}}$ which is the most similar to the collaborators' correlation matrix $C_{u_{1}, u_{2}}^{\left(t_{l}\right)}$, obtained for the pair of users $\left(u_{1}, u_{2}\right)$ at given timestamp $t_{l}$.

$$
\begin{aligned}
& \operatorname{argmin}_{R_{1,2}}\left\|F^{R_{1,2}} \ominus C_{u_{1}, u_{2}}^{\left(t_{l}\right)}\right\| \\
& \text { subject to : } \left.^{\forall_{\left(f_{j}, f_{k}\right) \in \mathcal{K}^{R_{1,2}}}} F^{R_{1,2}}\left(f_{j}, f_{k}\right)-C_{u_{1}, u_{2}}^{\left(t_{l}\right)}\left(f_{j}, f_{k}\right)\right)>-1
\end{aligned}
$$

where $\|$.$\| represents the Frobenius norm and \ominus$ is the minus operator defined as:

$$
F^{R_{1,2}}\left(f_{j}, f_{k}\right) \ominus C_{u_{1}, u_{2}}^{\left(t_{l}\right)}\left(f_{j}, f_{k}\right)=\left\{\begin{array}{c}
F^{R_{1,2}}\left(f_{j}, f_{k}\right) \ominus C_{u_{1}, u_{2}}^{\left(t_{l}\right)}\left(f_{j}, f_{k}\right) \\
\text { if } F^{R_{1,2}}\left(f_{j}, f_{k}\right) \in\{-1 ; 1\} \\
0 \quad \text { otherwise }
\end{array}\right.
$$

\subsubsection{Ground truth and Metrics}

We built the ground truth using clickthrough data following the assumption that implicit relevance derived from clicks is reasonably accurate (Joachims et al., 2005). Specifically, the ground truth only relies on the clicked documents and includes an agreement level, as suggested in 
(Shah and González-Ibáñez, 2011b). However, in contrast to Soulier et al. (2014), who consider an agreement level involving two users, we reinforce the agreement level condition by accounting for the bias of intra-group collaboration interactions via the notion that participants might belong to different groups. Indeed, collaborators are likely to interact through the chat system in order share document links, as suggested in Section 4.1.1. This results in a small relevant document set, namely 38 and 20 for user studies US1 and US2, respectively.

Using (Shah and González-Ibáñez, 2011b), we used well-known collaborative-based metrics proposed to evaluate the search outcomes of collaborative search. These metrics are precision- and recall-oriented, and are estimated at the group level. As such, they consider documents selected by collaborators throughout the search session in which submitted queries constitute a collective action, rather than independent actions. In order to evaluate the retrieval effectiveness of our proposed model, the metrics are applied to a document set that aggregates rankings retrieved throughout the session. These metrics are estimated at the group level (as done by Shah and González-Ibáñez (2011b)) across all queries submitted by all collaborators. In particular, we (a) considered rankings with respect to their top 20 ranked documents as usually done in the information retrieval domain by the rank $R$ (Buckley and Voorhees, 2000), (b) merged the top 20 documents of all rankings retrieved with respect to queries submitted throughout the search session by all collaborators, and then (c) estimated the collaborative metrics of this merged document set, namely at the group level.

In order to estimate the collaborative-based metrics, we adapted the universe, relevant universe, coverage and relevant coverage sets defined in (Shah and González-Ibáñez, 2011b):

- The universe $U$ of web pages represents the document dataset.

- The relevant universe $U_{r}$ refers to the ground truth, with $U_{r} \subset U$.

- The coverage $\operatorname{Cov}(g)$ of a collaborative group $g$ expresses the total number of distinct documents retrieved for all queries submitted by collaborators of group $g$ throughout the search session.

- The relevant coverage $\operatorname{Rel} \operatorname{Cov}(g)$ of a collaborative group $g$ refers to 
the total number of distinct relevant documents retrieved for all queries submitted by collaborators of group $g$ throughout the search session.

With this in mind, we used the following collaborative metrics to measure the synergic effect of a collaborative group $g$ :

- The precision $\operatorname{Prec}(g)$ estimated for collaborative group $g$ :

$$
\operatorname{Prec}(g)=\frac{\operatorname{RelCov}(g)}{\operatorname{Cov}(g)}
$$

- The recall Recall $(g)$ estimated for collaborative group $g$ :

$$
\operatorname{Recall}(g)=\frac{\operatorname{RelCov}(g)}{U_{r}}
$$

- The F-measure $F(g)$ estimated for collaborative group $g$ which combines both precision and recall metrics:

$$
F(g)=\frac{2 * \operatorname{Prec}(g) * \operatorname{Recall}(g)}{\operatorname{Prec}(g)+\operatorname{Recall}(g)}
$$

Finally, these measures are averaged over the collaborative groups of each user study, namely the 25 collaborative groups (US1) on one hand and the 10 (US2) on the other hand.

Note that the computation of these collaborative-oriented metrics is as similar as possible to the precision and recall measures in classical information retrieval. However, while classical IR considers a ranking an evidence source, we rely here on a set built by a merging of the top 20 documents retrieved for all queries submitted over the search session by all collaborators.

\subsection{Results}

This section reports the obtained results with respect to several scopes. First, we present the parameter tuning step and then, we analyze the retrieval effectiveness. 


\subsubsection{Parameter Tuning}

In order to highlight the consistency of our model regardless of specific users, tasks, and topics, we performed a learning-testing approach in two steps, illustrated in Figure 6: (1) the learning step, which optimizes the model parameter(s) using one of our datasets, e.g. US1, and (2) the testing step, which estimates the retrieval effectiveness of our model on the other dataset, e.g. US2, according to parameter optimal value(s) found in the learning step.

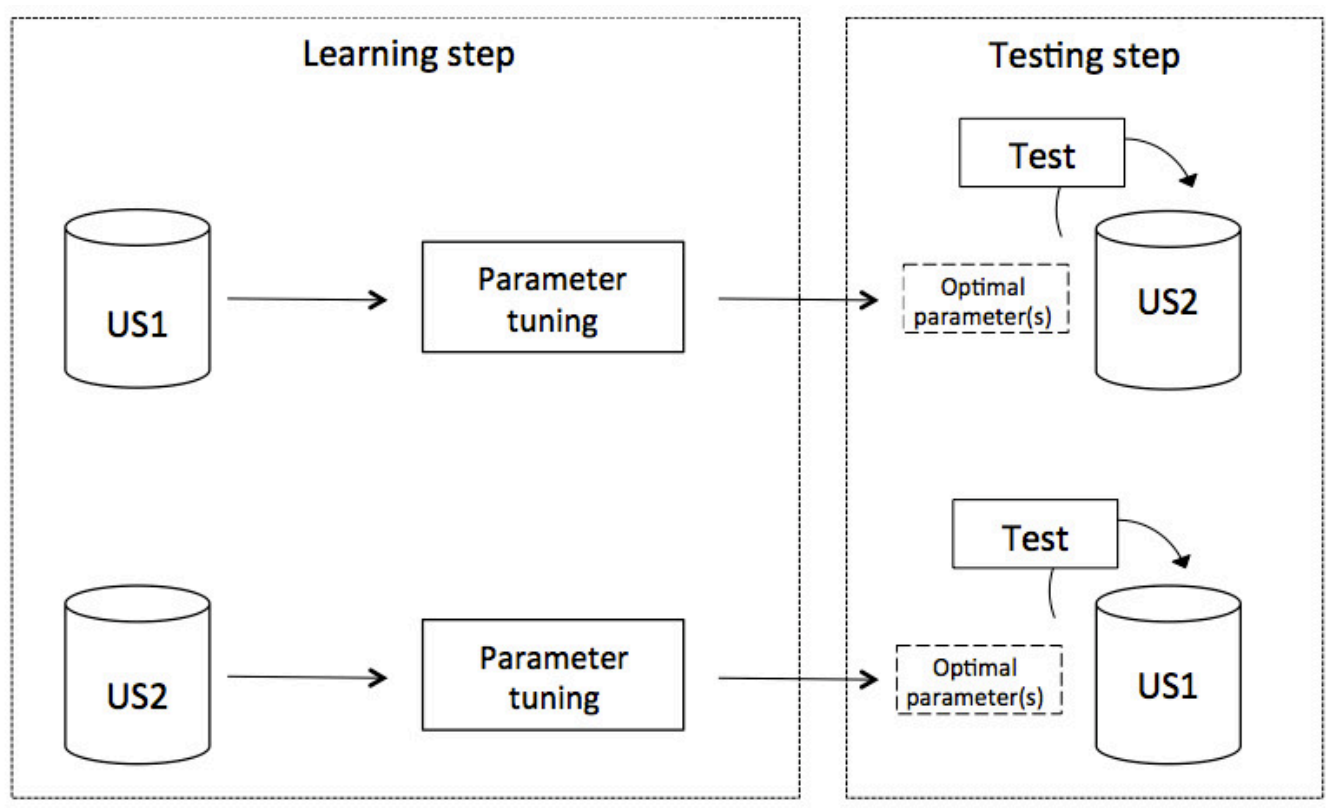

Figure 6: Parameter tuning methodology

Note that our model uses a feature set to dynamically mine collaborators' latent roles by leveraging features' importance within the collaborative ranking model and collaborators' complementarities. With this in mind, we expressed the assumptions that the decay parameter $\gamma$ combining these two aspects in the optimization problem (see Equation 4) might be fixed over the session. Accordingly, the tuning phase mainly concerns the $\gamma$ parameter, which expresses the collaborators' complementarity. Both versions of our proposed model, namely MineRank(q) and MineRank(t), are concerned with this tuning, and we consider the F-measure indicator to be the tuned 
effectiveness metric because it is a combination of precision and recall.

The first version of our model, namely MineRank(q), launched at each query submission only depends on the parameter $\gamma$, used within the latent role mining step (Equation 4). The latter was tuned with a value range $\gamma \in[0 . .1]$, as illustrated in Figure 7. We can see that the optimal value for parameter $\gamma$ is reached at 0.5 and 0.2 for, respectively, user studies US1 and US2, with an F-measure value respectively equal to 0.074 and 0.060 . This difference suggests that the constraint of the report-writing in US2 does not allow collaborators to fully emphasize their search behavior complementarity.

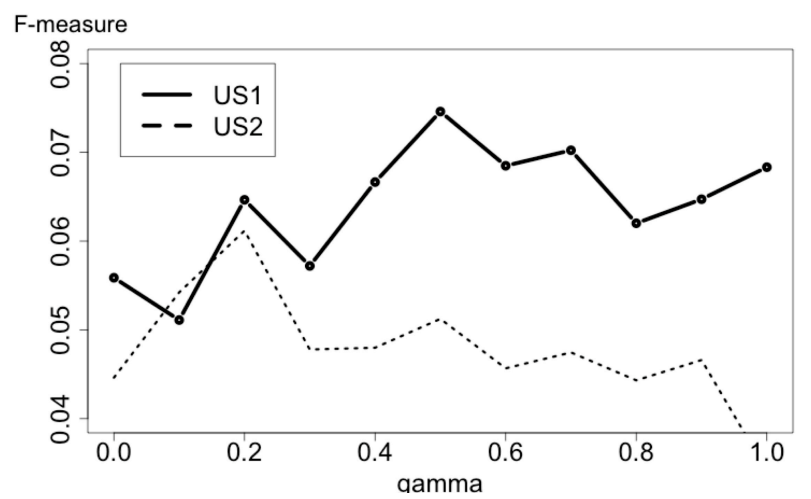

Figure 7: Parameter tuning of MineRank(q)

The second version of our model, namely MineRank(t), requires fixing the parameter $\gamma \in[0 . .1]$, as previously done, and also timestamp $t$ where the latent role mining step is launched. We consider that a 1-to-5-minute time window for mining latent roles is a reasonable range for our experiments. This better fits with our model's assumption that search behaviors evolve throughout the search session. Figure $8 \mathrm{a}$ and Figure $8 \mathrm{~b}$ illustrate the variation of the F-measure for our model MineRank(t) with respect to both parameters $\gamma \in[0 . .1]$ and $t \in[1 . .5]$, for, respectively, user studies US1 and US2. The F-measure is optimal $(F=0.069)$ when $\gamma=0.5$ and $t=2$ for dataset $U S 1$, while in dataset US2, it reaches 0.056 when $\gamma=0.1$ and $t=3$.

The optimal values $\gamma$ obtained for both versions highlight that the consideration of search skill complementarities within the role mining approach is 


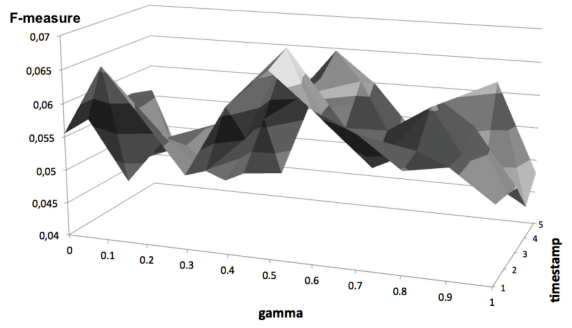

(a) MineRank(t) model for US1

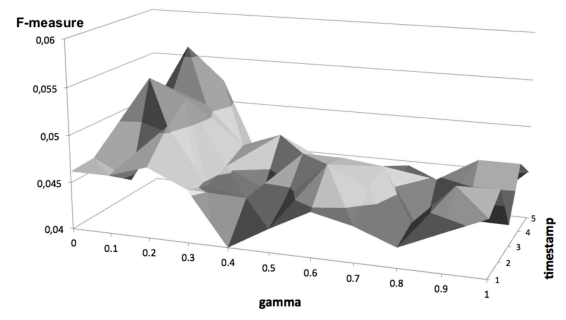

(b) MineRank(t) model for US2

Figure 8: Parameter tuning of MineRank(t)

higher in user study US1 than in user study US2. Moreover, scores obtained for both versions highlight that the effectiveness of the model MineRank(t) is lower than that of model MineRank(q) in both user studies. This is consistent with the fact that our model relies on relevance feedback expressed after each submitted query rather than after regular timestamps. Therefore, in the remaining experiments, we only consider the version $\operatorname{Mine} \operatorname{Rank}(q)$.

\subsubsection{Analyzing the dynamics of collaborators' latent roles}

In this section, our goal is to identify the evolution of search skills used by collaborators throughout the search session. For this purpose, we analyze, first, the average number of selected features for characterizing the latent role kernels of collaborators, and second, the average overlap between the feature set selected for two successive collaborators' latent role kernels. These indicators are estimated over time at each query submission, as illustrated in Figure 9. As a reminder, we call an iteration $t_{l}$ the time-window beginning at each time user $u$ submits query $q$ and ending when document list $\mathcal{D}_{u}^{t_{l}}$ is displayed to user $u$.

Since significant differences between features and a latent time interval are both required to highlight differences between collaborators (Soulier et al., 2014), we emphasize that only 3 collaborative groups from the 25 in user study US1 performed more than 60 iterations with respect to our model, and only one group performed more than 18 iterations in user study US2. Accordingly, the subsequently noticed variations are not significant.

From Figure 9a, respectively Figure 9b, we can see that the number of features increases over time and seems to reach an optimum between 8 and 


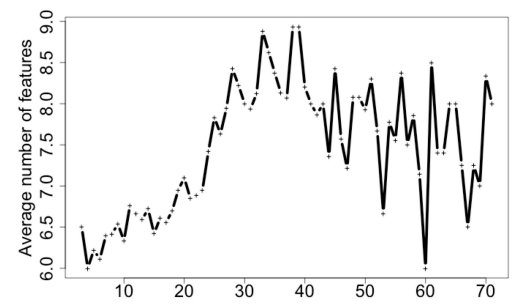

(a) Average number of features for user study US1

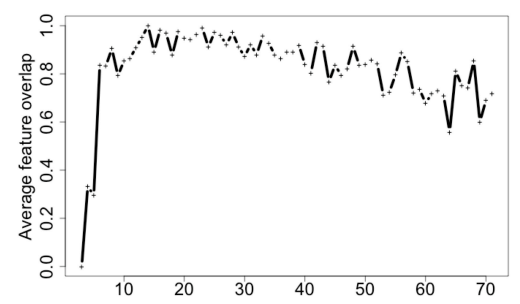

(c) Average feature set overlap for user study US1

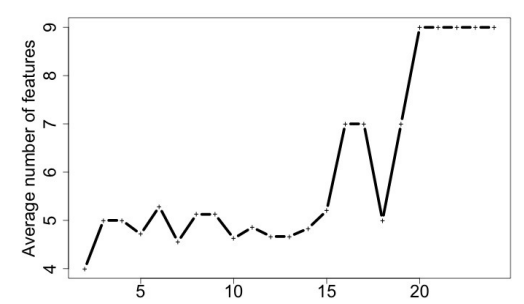

(b) Average number of features for user study US2

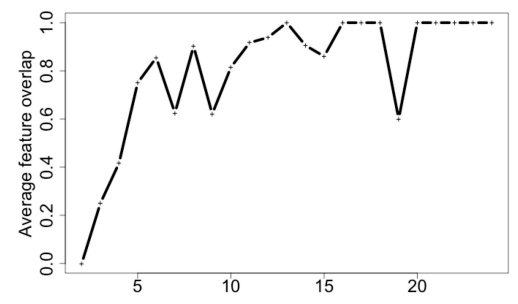

(d) Average feature set overlap for user study US2

Figure 9: Analysis of collaborators' latent role evolution throughout the whole search sessions.

9 , respectively 7 , search behavior features over the 11 for US1, respectively US2. First, the fact that the number of features increases over time might be explained by the fact that the amount of considered data (namely, relevance feedback) in the algorithm increases over time, thus increasing the likelihood that we obtain significant p-value within the collaborators' behavior differences analysis. Second, the difference between both datasets can be attributed to the participants' tasks, but could also suggest that a more intensive activity within the search process-through submitted queries, for instance-gives a better landscape of collaborators complementarities.

In Figure 9c and Figure 9d, the overlap indicator is also close to 1 after the $20^{t h}$ submitted query for both datasets, which indicates that the kernel of the latent roles mined after this timestamp is almost stable over the remainder of the session. This suggests that participants naturally adopt the best behaviors with respect to their search skills, which tend to converge and be 
persistent over the time. Beforehand, participants take time to identify their best search strategies, and the latent role varies between successive query submissions.

\subsubsection{Retrieval Effectiveness}

In this section, we measure the retrieval effectiveness of our collaborative ranking approach based on latent role mining $\operatorname{MineRank(q)}$ with respect to state-of-the-art ranking models BM25, Logit, GS, PM and RoleMining. Table 5 presents the obtained results.

Table 5: Comparison of the role mining impact on the retrieval effectiveness. \%Chg: MineRank improvement. Student test significance *: $0.01<t \leq 0.05$; $* *$ : $0.001<t \leq 0.01 ; * * *: t \leq 0.001$.

\begin{tabular}{|c|c|c|c|c|c|c|c|c|c|c|}
\hline \multirow{2}{*}{\multicolumn{2}{|c|}{ training set $\rightarrow$ testing set }} & \multicolumn{3}{|c|}{$\overline{P r e c @ 20}$} & \multicolumn{3}{|c|}{ Recall@20 } & \multicolumn{3}{|c|}{$F @ 20$} \\
\hline & & value & $\%$ Chg & $t$ & value & $\% C h g$ & $t$ & value & $\%$ Chg & $t$ \\
\hline \multicolumn{11}{|c|}{ Ground truth: Collaborative methodology } \\
\hline \multirow{6}{*}{$U S 2 \rightarrow U S 1$} & BM25 & 0,009 & 285,79 & **** & 0,066 & 236,51 & $* * *$ & 0,017 & 280,84 & *** \\
\hline & Logit & 0,031 & 20,66 & & 0,155 & 43,24 & * & 0,052 & 23,64 & \\
\hline & GS & 0,009 & 306,78 & *** & 0,045 & 400,10 & $* * *$ & 0,015 & 324,29 & $* * *$ \\
\hline & $\mathrm{PM}$ & 0,014 & 178,55 & *** & 0,029 & 650,15 & $* * *$ & 0,018 & 254,85 & *** \\
\hline & RoleMining & 0.012 & 217.82 & *** & 0.076 & 194.44 & $* * *$ & 0.020 & 217.99 & $* * *$ \\
\hline & MineRank(q) & 0,038 & & & 0,223 & & & 0,065 & & \\
\hline \multirow{6}{*}{$U S 1 \rightarrow U S 2$} & BM25 & 0,015 & 85,92 & * & 0,163 & 74.19 & $*$ & 0,027 & 85.29 & * \\
\hline & Logit & 0,025 & 10,67 & & 0,252 & 12,50 & & 0,046 & 10,92 & \\
\hline & GS & 0,030 & $-6,24$ & & 0,184 & 54.28 & & 0,051 & 0 & \\
\hline & $\mathrm{PM}$ & 0,056 & $-50,04$ & *** & 0,205 & 38.46 & * & 0,088 & -41.87 & $* * *$ \\
\hline & RoleMining & 0.024 & 18.91 & & 0.216 & 31.70 & $*$ & 0.046 & 20.13 & * \\
\hline & MineRank(q) & 0,028 & & & 0,284 & & & 0,051 & & \\
\hline
\end{tabular}

From a general point of view, we notice low evaluation metric values obtained using baselines as well as our proposed MineRank model. These low-level effectiveness results could be due to the association between different facts. Indeed, we hypothesize that precision measures are bounded de facto because of the small number of assumed relevant documents (see Section 4.1.4) for both user studies (38 and 20 for respectively $U S 1$ and US2). Moreover, since the SERPs were extracted asynchronously with participants' search task, as explained in section 4.1.2, the ground truth is more likely to be smaller since the documents clicked by the participants alongside the search task are not obviously in the top ranks of the SERPs. However, since the same evaluation process is carried out over the different 
models, we believe that the comparison between baselines and the proposed model is reasonable.

In Table 5, we can see that our model generally provides higher results than the four baselines with significant improvements ranged between $20.13 \%$ and $650.15 \%$. Significantly, the improvements with respect to the recall measure are generally higher than those obtained for the precision measure, which suggests that our model is more recall-oriented. Indeed, this statement seems realistic given that the evaluation metrics are estimated at the group level, and thus aggregate the different lists retrieved for all collaborators. Therefore, the coverage is more likely to be higher than the size of the ground truth, leading recall measures to usurp precision measures. Moreover, for user study US1, our model significantly exceeds both individual and collaborative baselines from $43.24 \%$ to $650.15 \%$ over the three metrics. For the second user study US2, we obtained significant improvements for the BM25 and RoleMining baselines over at least two metrics as well as the collaborative ranking models $P M$ for the recall measure. We note, however, that our model MineRank $(q)$ seems to be less effective than GS and PM baselines for the second user study US2 in terms of precision and F-measure. We will address this issue later in the analysis.

In summary, these results highlight four main contributions:

1. Our model enables users to benefit from the synergic effect of collaboration since the effectiveness of the collaboration (our model MineR$a n k$ ) is greater than the sum of the individual search sessions (baseline BM25);

2. Ranking documents with respect to latent roles gives an additional value to a CIR model based only on the behavior analysis of collaborators, especially in terms of recall (baseline Logit);

3. Mining latent roles for collaborators seems to be more effective than a CIR scenario in which roles are fixed throughout the search session (baselines $G S$ and $P M$ );

4. Leveraging complementarity in unlabeled roles of collaborators seems more effective than mining predefined roles with respect to their differences in search behaviors (baseline RoleMining). 
However, by comparing both datasets, we observe that improvements are lower for user study US2, particularly with respect to the $P M$ baseline for the precision measure, and accordingly the F-measure. This can be explained by two main reasons. First, the ratio between the number of submitted queries and the size of the document collection of both user studies suggests that the US1 dataset seems to be more topic-concentrated than US2. Another reason might be that the observed difference in retrieval effectiveness between US1 and US2 is due to the difference in the ability of the latent role kernel, identified using Coll-Clique algorithm on both datasets to the capture complementarities between the involved users. To investigate this hypothesis, we compared, for each user study, (1) the kernel tuned with the optimal setting $M_{O}$ ( $\gamma=0.5$ resp. 0.2 for US1 and US2 achieving the optimal retrieval effectiveness based on F-measure, as shown in Figure 7), and (2) the kernel identified in the tested model MineRank(q). More precisely, we carried out a statistical analysis in order to determine, for each model $\left(M_{O}\right.$ and MineRank $\left.(q)\right)$, which features impacted the F-measure. For this purpose, we performed an ANOVA analysis between the F-measure obtained at each query submission and the whole features, noted as binary indicators in reference to the presence or absence of the features within the kernel of the mined latent role. To obtain the best model, we first consider a full model including all explanatory variables (binary indicators of features), and then performed a backward elimination to iteratively remove the less significant factors with respect to the F-measure.

Table 6 presents the obtained models after the backward elimination for both models MineRank $(q)$ and $M_{O}$ and both user studies US1 and US2. We can see that, for both MineRank $(q)$ and $M_{O}$, the set of significant features is larger for user study US1 than for US2, as can be expected from Figure 9. This suggests that there is a wider range of differences between collaborators in US1 than in US2, allowing them to fully benefit from the collaborators' skills complementarity. Moreover, comparing the best explicative models obtained for MineRank $(q)$ with $M_{O}$, we can see that for US1, half $(3 / 6)$ of the features highlighted as significant for $M_{O}$ are also significant for MineRank(q), while for US2 any (0/3) significant features for $M_{O}$ have been highlighted as significant for MineRank $(q)$.

Combining these observations, we can clearly explain the low socres obtained for US2. Indeed, the difference between the number of significant 
Table 6: Explanatory features for the F-measure according to the ANOVA statistical analysis. p-value *: $0.01<p \leq 0.05$; **: $0.001<p \leq 0.01$; ***: $p \leq 0.001$.

\begin{tabular}{lllll}
\hline & \multicolumn{2}{c}{ MineRank $(q)$} & \multicolumn{2}{c}{$M_{O}$} \\
& Feature & p-value & Feature & p-value \\
\hline \multirow{4}{*}{ UiO } & $0.033^{*}$ & $\mathrm{TiO}$ & $0.002^{* *}$ \\
& $\mathrm{Spec}$ & $0.050^{*}$ & $\mathrm{TeO}$ & $0.034^{*}$ \\
& $\mathrm{SO}$ & $0.033^{*}$ & $\mathrm{TQTP}$ & $0.006^{* *}$ \\
& & & $\mathrm{VP}$ & $0.000^{* * *}$ \\
& & & $\mathrm{Spec}$ & $0.015^{*}$ \\
& & & $\mathrm{SO}$ & $0.000^{* * *}$ \\
\hline \multirow{3}{*}{$\mathrm{US2}$} & $\mathrm{TOD}$ & $0.032^{*}$ & $\mathrm{TiO}$ & $0.036^{*}$ \\
& & & $\mathrm{TeO}$ & $0.001^{* * *}$ \\
& & & $\mathrm{VP}$ & $0.021^{*}$ \\
\hline
\end{tabular}

features in the MineRank setting and in the $M_{O}$ one, resulting in a single feature $(T O D)$, suggests that this set of features is insufficient for modeling behavioral differences/complementarities between users. This could be explained by the fact that the Coll-Clique algorithm is based on the $\gamma$ parameter tuned on users from US1, who behave differently.

\section{Discussion and Conclusion}

While several works claim that roles structure collaboration among participants (Kelly and Payne, 2013), some role-based approaches inadequately leverage the skills that individuals bring to a collaboration, as well as the dynamics of a collaboration. Recently, a new approach (Soulier et al., 2014) combining a user-driven and a system-based mediation has been proposed, making it possible to leverage collaborators' search behaviors in order to mine predefined roles belonging to a taxonomy.

In this article, we presented an unsupervised method to mine collaborators' latent roles on the basis of their evolving search behavior complementarities. These latent roles, and more particularly the search features identified for characterizing their latent roles, are re-injected within the collaborative document ranking. Experiments on two user studies highlight that collaborators are able to benefit from the synergic effect 
of the collaboration while the real-time mining of their search behavior complements their latent roles.

However, this work is not without limitations that could lead to different perspectives.

First, we focus on the model design, which relies on a particular collaborative setting between a pair of synchronously working users. We believe that our model could be enhanced as follows.

- Some work has found that collaboration is generally performed in larger groups (Morris, 2013), and we believe that our contribution would mature if it could facilitate collaboration between more than two users. This extension would raise new challenges in the latent role modeling as well as the model steps. In particular, a single correlation matrix is not sufficient to highlight search skill differences and similarities between users in a larger group since it is adapted for a dyad. Because our intuition is to identify in which skill each user is the most effective, an intrinsic analysis of search behaviors would lack the principles of division of labor and sharing of knowledge that guide collaboration. With this in mind, one solution could be to first build latent roles pair by pair, which could then be aggregated and analyzed at the group level in order to identify each collaborator's skills.

- Also, the synchronicity of the search actions could be considered differently assuming that users could work at different time-windows. This temporal asynchronicity raises the issue of multi-session management, which could impact the search behavior modeling based on cumulative values of search features from the beginning of the search session to a given timestamp.

- Furthermore, we assumed that collaborators have complementary skills (Sonnenwald, 1996), which is not always the case in a CIR setting. For instance, in the retrieval model proposed by (Foley and Smeaton, 2009), document rankings are not personalized, which implies that the complementarity between collaborators is not necessarily required.

Second, experiments are somehow restricted to a particular framework which could limit the generalizability of our results. We highlight its peculiarities below. 
- We evaluated our model through user studies in which participants aimed to solve an exploratory task. We highlight that other types of tasks are also used in collaborative search, such as travel planning, shopping, fact-finding tasks, etc (Morris, 2013). Therefore, a future study would analyze whether the latent role would flourish in these other types of tasks, which are more practical and less topic-oriented, and in which search skills are predominant.

- Last, our experimentation consists of a log-study in which latent role mining and collaborative document ranking are performed on search logs. We are aware that a user-study would be a more appropriately fit for tracking collaborators' search behaviors (interactions, communications, or search strategies guided by ranking retrieved by the proposed model), but log-study-based evaluation protocol is less time-consuming and enables us to compare different baselines without additional costs. We emphasize, however, that search logs are those collected during a real collaborative search session, thus making our evaluation as natural as possible. In the near future, we plan to evaluate our model through a user-study-oriented evaluation in which participants interact with a system that supports the proposed CIR model based on collaborators' latent roles. We assume that this interactive and real-time user-study would also tackle the low metric value issue that we point out during the experiments.

\section{Appendix A. Coll-Clique illustration}

In order to illustrate our algorithm, we consider a collaborative search setting involving two users $u_{1}$ and $u_{2}$ whose behavioral activity is modeled by a set $F$ of features $\left\{f_{1}, f_{2}, f_{3}, f_{4}\right\}$.

Figure A.10 illustrates the feature-based graph representing the behavior of these two collaborators $u_{1}$ and $u_{2}$ at timestamp $t_{l}$. For instance, the weight of node $f_{1}$, namely 0.84 , represents the feature importance $f_{1}$ estimated by Equation 2. The weight of the edge connecting $f_{1}$ and $f_{4}$ expresses the correlation between the collaborators' differences towards these two features, also perceived as the level of complementarity $C_{1,2}^{\left(t_{l}\right)}\left(f_{1}, f_{4}\right)$.

Table A.7 presents our Coll-Clique algorithm relying on the behavioral graph presented in Figure A.10. The "Rec." column expresses the degree of 


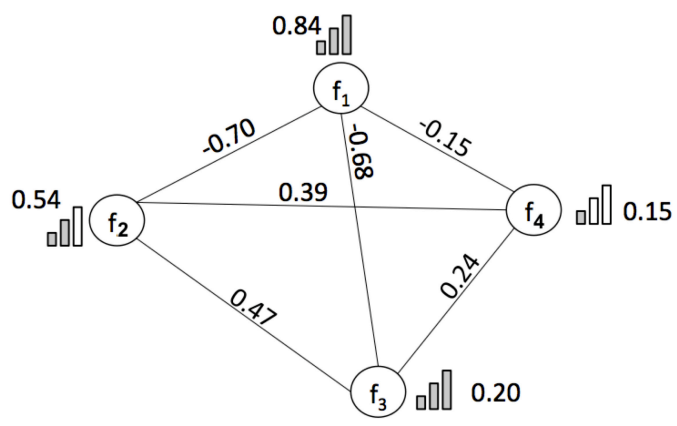

Figure A.10: Example of graph modeling collaborators behaviors according to four behavioral features

the recursion in the algorithm. Columns "C", "P" and "K" represent the recursion input data while columns "C $\oplus f_{h}$ ", "P" and " $K_{\text {end }}$ " represent the outputs of the recursion, respectively including the growing clique, the new candidate network in which feature weight is depreciated, and the current maximum clique.

At the end of the recursion, the local clique consists of the feature set including $f_{1}, f_{2}$, and $f_{3}$ since the sum of the node weight $W(C)$ is greater than the weight $W(K)$ of current clique $K$.

\section{Appendix B. Aknowledgements}

We thank the reviewers for their valuable remarks. We thank participants of user studies for their involvement. 


\begin{tabular}{|c|c|c|c|c|c|c|c|c|c|}
\hline Rec. & $\mathrm{C}$ & $\mathrm{P}$ & $\mathrm{K}$ & $\mathrm{W}(\mathrm{C})$ & $\mathrm{W}(\mathrm{P})$ & $\mathrm{W}(\mathrm{K}$ & $C \oplus f_{h}$ & $\mathrm{P}^{\prime}$ & $K_{\text {end }}$ \\
\hline \multirow[t]{4}{*}{ init } & \{\} & $1(0.84)$ & \{\} & 0 & 1.73 & 0 & - & - & - \\
\hline & & $2(0.54)$ & & & & & & & \\
\hline & & $3(0.20)$ & & & & & & & \\
\hline & & $4(0.15)$ & & & & & & & \\
\hline \multirow[t]{4}{*}{1} & \{\} & $1(0.84)$ & \{\} & 0 & 1.73 & 0 & $1(0.84)$ & $2(1.24)$ & - \\
\hline & & $2(0.54)$ & & & & & & $3(0.88)$ & \\
\hline & & $3(0.20)$ & & & & & & $4(0.3)$ & \\
\hline & & $4(0.15)$ & & & & & & & \\
\hline \multirow[t]{3}{*}{1.1} & $1(0.84)$ & $2(1.24)$ & \{\} & 0.84 & 2.42 & 0 & $1(0.84)$ & $3(0.41)$ & - \\
\hline & & $3(0.88)$ & & & & & $2(1.24)$ & & \\
\hline & & $4(0.3)$ & & & & & & & \\
\hline \multirow[t]{3}{*}{1.1 .1} & $1(0.84)$ & $3(0.41)$ & \{\} & 2.08 & 0.41 & 0 & $1(0.84)$ & \{\} & $1(0.84)$ \\
\hline & $2(1.24)$ & & & & & & $2(1.24)$ & & $2(1.24)$ \\
\hline & & & & & & & $3(0.41)$ & & $3(0.41)$ \\
\hline \multirow[t]{3}{*}{1.1} & $1(0.84)$ & $2(1.24)$ & $1(0.84)$ & 0.84 & 2.42 & 2.49 & $1(0.84)$ & $2(0.07)$ & $1(0.84)$ \\
\hline & & $3(0.88)$ & $2(1.24)$ & & & & $3(0.88)$ & $4(0.06)$ & $2(1.24)$ \\
\hline & & $4(0.3)$ & $3(0.41)$ & & & & & & $3(0.41)$ \\
\hline \multirow[t]{3}{*}{1.1 .1} & $1(0.84)$ & $2(0.07)$ & $1(0.84)$ & 1.72 & 0.13 & 2.53 & STOP & \multirow{3}{*}{\multicolumn{2}{|c|}{ ALGORITHM }} \\
\hline & $3(0.88)$ & $4(0.06)$ & $2(1.24)$ & & & & & & \\
\hline & & & $3(0.41)$ & & & & & & \\
\hline
\end{tabular}

Table A.7: Illustration of a recursion of our Coll-Clique algorithm

\section{References}

Agichtein, E., Brill, E., Dumais, S., Ragno, R., 2006. Learning user interaction models for predicting web search result preferences, in: SIGIR, pp. $3-10$.

Bennett, P.N., White, R.W., Chu, W., Dumais, S.T., Bailey, P., Borisyuk, F., Cui, X., 2012. Modeling the impact of short- and long-term behavior on search personalization, in: SIGIR, pp. 185-194.

Buckley, C., Voorhees, E.M., 2000. Evaluating evaluation measure stability, in: Proceedings of the 23rd Annual International ACM SIGIR Conference on Research and Development in Information Retrieval, ACM. pp. 33-40.

Carraghan, R., Pardalos, P.M., 1990. An exact algorithm for the maximum clique problem. Operations Research Letters 9, 375 - 382. 
Dourish, P., Bellotti, V., 1992. Awareness and coordination in shared workspaces, in: Proceedings of the ACM Conference on Computersupported Cooperative Work, ACM. pp. 107-114.

Evans, B.M., Chi, E.H., 2010. An elaborated model of social search. Inf. Process. Manage. 46, 656-678.

Fidel, R., Bruce, H., Pejtersen, A.M., Dumais, S.T., Grudin, J., Poltrock, S., 2000. Collaborative Information Retrieval (CIR). The New Review of Information Behaviour Research 1, 235-247.

Foley, C., Smeaton, A.F., 2009. Synchronous collaborative information retrieval: Techniques and evaluation, in: ECIR, pp. 42-53.

Foley, C., Smeaton, A.F., 2010. Division of labour and sharing of knowledge for synchronous collaborative information retrieval. Inf. Process. Manage. $46,762-772$.

Geng, X., Liu, T.Y., Qin, T., Li, H., 2007. Feature selection for ranking, in: SIGIR, pp. 407-414.

Golder, S.A., Donath, J., 2004. Social roles in electronic communities, in: Proc. of Association of Internet Researchers Conference.

Golovchinsky, G., Qvarfordt, O., Pickens, J., 2009. Collaborative information seeking. IEEE Computer 42, 47-51.

Heath, A.P., White, R.W., 2008. Defection detection: Predicting search engine switching, in: WWW, pp. 1173-1174.

Henderson, K., Gallagher, B., Eliassi-Rad, T., Tong, H., Basu, S., Akoglu, L., Koutra, D., Faloutsos, C., Li, L., 2012. Rolx: Structural role extraction \& mining in large graphs, in: SIGKDD, pp. 1231-1239.

Joachims, T., 2002. Optimizing search engines using clickthrough data, in: SIGKDD, pp. 133-142.

Joachims, T., Granka, L.A., Pan, B., Hembrooke, H., Gay, G., 2005. Accurately interpreting clickthrough data as implicit feedback, in: SIGIR, ACM. pp. 154-161. 
Joho, H., Hannah, D., Jose, J.M., 2009. Revisiting ir techniques for collaborative search strategies, in: ECIR, pp. 66-77.

Kelly, R., Payne, S.J., 2013. Division of labour in collaborative information seeking: Current approaches and future directions, in: CIS Workshop at CSCW.

Kwak, H., Lee, C., Park, H., Moon, S., 2010. What is twitter, a social network or a news media?, in: WWW, pp. 591-600.

Leung, K.W.T., Lee, D.L., Ng, W., Fung, H.Y., 2008. A framework for personalizing web search with concept-based user profiles. ACM Trans. Internet Technol. 11, 17:1-17:29.

McCallum, A., Wang, X., Corrada-Emmanuel, A., 2007. Topic and role discovery in social networks with experiments on enron and academic email. J. Artif. Int. Res. 30, 249-272.

Morris, M.R., 2013. Collaborative Search Revisited, in: Proceedings of the Conference on Computer Supported Cooperative Work, ACM. pp. 11811192.

Morris, M.R., Horvitz, E., 2007. Searchtogether: an interface for collaborative web search, in: UIST, pp. 3-12.

Morris, M.R., Teevan, J., Bush, S., 2008. Enhancing collaborative web search with personalization: groupization, smart splitting, and group hithighlighting, in: CSCW, pp. 481-484.

Pal, A., Counts, S., 2011. Identifying topical authorities in microblogs, in: WSDM, pp. 45-54.

Pickens, J., Golovchinsky, G., Shah, C., Qvarfordt, P., Back, M., 2008. Algorithmic mediation for collaborative exploratory search, in: SIGIR, pp. $315-322$.

Radinsky, K., Svore, K.M., Dumais, S.T., Shokouhi, M., Teevan, J., Bocharov, A., Horvitz, E., 2013. Behavioral dynamics on the web: Learning, modeling, and prediction. ACM Trans. Inf. Syst. 31, 16:1-16:37. 
Robertson, S.E., Walker, S., 1994. Some simple effective approximations to the 2-Poisson model for probabilistic weighted retrieval, in: Proceedings of the Annual International SIGIR Conference on Research and Development in Information Retrieval, ACM. pp. 232-241.

Shah, C., González-Ibáñez, R., 2011a. Coagmento - A System for Supporting Collaborative Information Seeking., in: Demo in Proceedings of Association for Information Science and Technology Annual Meeting, pp. 9-12.

Shah, C., González-Ibáñez, R., 2011b. Evaluating the synergic effect of collaboration in information seeking, in: SIGIR, pp. 913-922.

Shah, C., Pickens, J., Golovchinsky, G., 2010. Role-based results redistribution for collaborative information retrieval. Inf. Process. Manage. 46, $773-781$.

Sonnenwald, D.H., 1996. Communication roles that support collaboration during the design process. Design Studies 17, 277-301.

Soulier, L., Shah, C., Tamine, L., 2014. User-driven system-mediated collaborative information retrieval, in: SIGIR, pp. 485-494.

Soulier, L., Tamine, L., Bahsoun, W., 2013. A collaborative document ranking model for a multi-faceted search, in: AIRS, pp. 109-120.

Tamine, L., Soulier, L., 2015. Understanding the impact of the role factor in collaborative information retrieval, in: Proceedings of the 24th ACM International on Conference on Information and Knowledge Management, pp. $43-52$.

Teevan, J., Dumais, S.T., Horvitz, E., 2005. Personalizing search via automated analysis of interests and activities, in: SIGIR, pp. 449-456.

Twidale, M.B.T., Nichols, D.M.N., Paice, C.D., 1997. Browsing is a Collaborative Process. Inf. Process. Manage. 33, 761-783.

White, R.W., Dumais, S.T., 2009. Characterizing and predicting search engine switching behavior, in: CIKM, pp. 87-96.

Wu, Q., Hao, J.K., 2015. A review on algorithms for maximum clique problems. European Journal of Operational Research 242, 693 - 709. 
Yue, Z., Han, S., He, D., 2014. Modeling search processes using hidden states in collaborative exploratory web search, in: CSCW, ACM. pp. 820-830. 